\title{
A Finite Element Computation of the Gravitational Radiation emitted by a Point-like object orbiting a Non-rotating Black Hole
}

\author{
Carlos F. Sopuerta ${ }^{1}$ and Pablo Laguna ${ }^{1,2}$ \\ ${ }^{1}$ Institute for Gravitational Physics and Geometry and Center for Gravitational Wave Physics, \\ Department of Astronomy \& Astrophysics, \\ The Pennsylvania State University, University Park, PA 16802, USA \\ ${ }^{2}$ Department of Physics, The Pennsylvania State University, University Park, PA 16802, USA
}

(Dated: September 13, 2018)

\begin{abstract}
The description of extreme-mass-ratio binary systems in the inspiral phase is a challenging problem in gravitational wave physics with significant relevance for the space interferometer LISA. The main difficulty lies in the evaluation of the effects of the small body's gravitational field on itself. To that end, an accurate computation of the perturbations produced by the small body with respect the background geometry of the large object, a massive black hole, is required. In this paper we present a new computational approach based on Finite Element Methods to solve the master equations describing perturbations of non-rotating black holes due to an orbiting point-like object. The numerical computations are carried out in the time domain by using evolution algorithms for wave-type equations. We show the accuracy of the method by comparing our calculations with previous results in the literature. Finally, we discuss the relevance of this method for achieving accurate descriptions of extreme-mass-ratio binaries.

PACS numbers: 04.30.Db, 04.40.Dg, 95.30.Sf, 97.10.Sj
\end{abstract}

\section{INTRODUCTION}

Extreme-Mass-Ratio Binaries (EMRBs) in the inspiral stage of their evolution are considered to be a primary source of gravitational radiation [1, 2] to be detected by the proposed laser interferometric space antenna LISA [3, 4, 5, 6]. They consist of a "small" object, such a main sequence star, a stellar mass black hole, or a neutron star, with mass $m$ ranging from $1 M_{\odot}$ to $10^{2} M_{\odot}$, orbiting a massive black hole (MBH) with mass $M$ ranging from $10^{3} M_{\odot}$ (if we consider the case of intermediate mass black holes in globular clusters) to $10^{9} M_{\odot}$ (the case of big supermassive black holes sitting in the center of galaxies). This translates to EMRBs with mass ratios, $\mu=m / M$, in the range $10^{-1}-10^{-9}$. In order to exploit this type of systems through LISA, it is crucial to have a good theoretical understanding of their evolution, good enough to produce accurate waveform templates in support of data analysis efforts. Because there is no significant coupling between the strong curvature effects produced by the MBH and its companion, relativistic perturbation theory is a well suited tool to study EMBRs. Clearly, the accuracy of this approximation depends on the smallness of the mass ratio $\mu$.

The challenge in modeling EMRBs is to compute the perturbations generated by the small body in the (background) gravitational field of the $\mathrm{MBH}$, and how these perturbations affect the motion of the small body itself. This problem has been known in literature as the radiation reaction problem. This is an old problem and several approaches to deal with it have been proposed (see the recent review by Poisson [7] and the contributions to [8]). The most extended approach consists in modelling the small object by using a point-like description and then, to describe the radiation reaction effects on the dynam- ics as the action of a local self-force that is responsible for the deviations from geodesic motion. A consistent derivation of the equations of motion coming out from this set up was given by Mino, Sasaki and Tanaka [9], and later, adopting an axiomatic approach, by Quinn and Wald 10 (see also 11]). However, these works only provide a formal prescription for the description of the orbital motion. For the practical calculations of the selfforce some techniques have been proposed: the mode-sum scheme 12, 13, 14, 15], and a regularization scheme based on zeta-function regularization techniques [16] (see 17] for a recent progress report).

The computation of the self-force and waveforms, and any other physical relevant information related to the inspiral due to radiation reaction constitute the main challenge of this problem. One possible way is to resort to analytic techniques by adding extra approximations to the problem, similar to those from post-Newtonian methods. However, the results may not be applicable to situations of physical relevance involving highly spinning MBHs and very eccentric orbits. To make computations without making further simplifications of the problem, numerical techniques appear to be a necessary tool. It is important to distinguish between frequency-domain and time-domain calculations. The frequency domain approach has been used for a long time; it provides accurate results for the computation of quasinormal modes and frequencies [18, 19]. However, the frequency-domain approach has more difficulties when we are interested in computing the waves originated from highly eccentric orbits since one has to sum over a large number of modes to obtain a good accuracy. In this sense, calculations in the time-domain can be more efficient for obtaining accurate waveforms for the physical situations of relevance.

However, the time-domain numerical approach has to 
face a challenge, which consists of dealing with the different physical scales (both spatial and temporal) present in the problem and that expand over several orders of magnitude. Specifically, one needs to handle not only large wavelength scales comparable to the massive black hole, but also to resolve the scales in the vicinity of the small object where radiation reaction effects play a crucial role. The conclusion is that we need to incorporate adaptive schemes in our numerical algorithms in order to provide the resolution that every region in the physical domain requires. Since the small object is going to be moving through the domain (unless we choose a very particular coordinate system), it is convenient to allow the adaptive scheme to change in time to distribute properly the resolution. Our choice to deal with these issues is the Finite Element Method (FEM), which is a numerical technique where adaptivity can be implemented in a natural way. The FEM has other properties, which we will discuss in this paper, that make it very suitable to be used for the description of EMRBs and also for other physical systems that are the subject of investigation in Numerical Relativity. In a recent work [20], we have already tested Adaptive Mesh Refinement techniques intrinsic to the FEM in a toy model consisting of a particle orbiting a black hole in the context of scalar gravity, where we have shown how an adaptive scheme can provide better accuracy than a non-adaptive scheme with an equivalent computational cost.

In this paper we use the FEM to perform time-domain simulations of a point-like object orbiting in geodesics (no radiation-reaction) around a non-rotating $\mathrm{MBH}$, and compute physically relevant quantities like energy and angular momentum emitted in gravitational waves and waveforms. This type of calculations constitute a good touchstone to evaluate the Finite Element (FE) techniques that we present in this paper, specially in relation to use this type of computations for the evaluation of the self-force on the particle. Since the MBH is nonrotating $\mathrm{MBH}$ the problem can be reduced to solve the one-dimensional Partial Differential Equations (PDEs) of black hole perturbations theory. These equations, in the Regge-Wheeler gauge, reduce to a master equation from which the metric perturbations can be fully recovered. The master equation for axial modes is known as the Regge-Wheeler equation, and for polar modes as the Zerilli-Moncrief equation. In this paper, instead of using the Regge-Wheeler function, we use a modification originally proposed by Cunningham, Price and Moncrief 21] that puts the axial modes on an equal footing with polar modes, as described by the Zerilli-Moncrief function, in relation to computing energy and angular momentum luminosities, and waveforms.

The plan of the paper is the following: In section 1 we summarize the main results from (non-rotating) blackhole perturbation theory that we need in our computations, including the explicit form of the source terms coming from the particle energy-momentum tensor. As far as we know, the expressions we present here for the sources associated with the axial modes, described by the Cunningham-Price-Moncrief master function, are new. We also perform an analysis of the discontinuities in the master equations due to the Dirac delta distributions that the source terms exhibit. In section [III we describe the numerical framework. We use a FE discretization for the spatial domain and a Finite Difference discretization in time. We start with the discretization of the domain, consisting of dividing the computational domain into disjoint subdomains (the elements). Then, we describe the FE functional spaces, which are finite-dimensional functional spaces used to approximate locally (at each element) our solution. The next step is the derivation of the weak form of the master equations, which is an integral form. It is important to remark that FE algorithms are derived from the integral form of the equations, in contrast with other numerical techniques where the differential form is used to obtain a discretization. From the weak form, we obtain the spatial discretization by imposing the vanishing of the residuals of our equations, which basically means to impose the vanishing of the components of the equations with respect to a basis of functions constructed from the FE functional spaces. This process leads to a coupled system of Ordinary Differential Equations (ODEs) which has a close analogy with the equations governing the behaviour of a system of coupled oscillators. A very important point in the discretization process is the fact that, because the $\mathrm{FE}$ formulation is based on an integral form of the equations, we obtain automatically a discretization of the sources containing Dirac delta distributions and its first derivative without having to resort to sequences of functions approaching in some limit the Dirac delta, we just use the properties of these distributions at the analytic level in the weak form of the equations. To solve the resulting ODEs we introduce a collection of evolution algorithms to solve the equations in second-order form and which have parameters that allow us to control the appearance of spurious high-frequency modes, which are common in systems like the one we are studying having a very localized source. We finish this section by discussing the structure of the mesh, in particular how adaptivity is implemented and how we can change in time this structure as the particle moves. In section [V] we discuss the performance of the FE numerical code we have developed and compare results regarding the computations of energy and angular momentum radiated with previous works in the literature, showing in this way the accuracy that this method is able to achieve. We conclude in section $\nabla$ where we discuss the convenience of using the FEM for the simulations of EMRBs in the light of the results of this paper and describe possible ways to proceed in the future to make this goal a reality. Finally, we have included two appendices: In Appendix $\mathrm{A}$ we summarize the geodesic equations of motion for the particle, and in Appendix B we briefly describe the Gauss-Legendre quadrature method for evaluating numerically some of the integrals that appear in the FE discretization of our 
equations.

The conventions that we follow throughout this work are: Greek letters are used to denote spacetime indices; capital Latin letters are used for indices in the time-radial part of the metric; lower-case Latin indices are used for the spherical sector of the metric. We use physical units in which $G=c=1$.

\section{SUMMARY OF PERTURBATION THEORY FOR NON-ROTATING BLACK HOLES}

Perturbation theory of black holes has a long history. It goes back to the seminal work by Regge and Wheeler 22 and later by Vishveshwara, Zerilli and Moncrief [18, 23, 24 for non-rotating black holes and to Teukolsky [25, 26] for rotating ones. At present, in the case of non-rotating black holes the metric perturbative scheme is completely developed and well understood at the linear level (see 27, 28, 29, 30, 31, 32, 33] for reviews). In the particular case of perturbations induced by an orbiting point-like object, which is the situation we are interested in this paper, there are a number of works on it 32, 34, 35, 36, 37, 38, 39]. Here, we summarize the theory using a particular formulation that makes the different expressions involved compact and self-explanatory.

We start from the perturbative splitting of the metric into the background, the non-rotating Schwarzschild black hole metric $\mathrm{g}_{\alpha \beta}^{\text {Sch }}$, and the small deviations $h_{\alpha \beta}$ :

$$
\mathrm{g}_{\alpha \beta}=\mathrm{g}_{\alpha \beta}^{\mathrm{Sch}}+h_{\alpha \beta} .
$$

Due to the spherical symmetry, the background manifold is the warped product $M^{2} \times S^{2}$, where $S^{2}$ denotes the 2-sphere and $M^{2}$ a two-dimensional Lorentzian manifold. The metric can then be written as the semidirect product of a Lorentzian metric on $M^{2}, \mathrm{~g}_{A B}$, and the unit curvature metric on $S^{2}$, that we call $\gamma_{a b}$ :

$$
\mathrm{g}_{\alpha \beta}=\left(\begin{array}{cc}
\mathrm{g}_{A B} & 0 \\
0 & r^{2} \gamma_{a b}
\end{array}\right) .
$$

Hereafter, $x^{A}$ denotes a coordinate system on $M^{2}$ and $x^{a}$ a coordinate system on $S^{2} ; r=r\left(x^{A}\right)$ is a function on $M^{2}$ that coincides with the invariantly defined radial area coordinate. In Schwarzschild coordinates we have

$$
\mathrm{g}_{A B} d x^{A} d x^{B}=-f d t^{2}+f^{-1} d r^{2}, \quad f=1-2 M / r .
$$

A vertical bar is used to denote the covariant derivative on $M^{2}$ and a semicolon to denote the one on $S^{2}$, thus we have $\mathrm{g}_{A B \mid C}=\gamma_{a b: c}=0$. We can also introduce the completely antisymmetric covariant unit tensors on $M^{2}$ and on $S^{2}, \epsilon_{A B}$ and $\epsilon_{a b}$ respectively, in such a way they satisfy: $\epsilon_{A B \mid C}=\epsilon_{a b: c}=0, \epsilon_{A C} \epsilon^{B C}=-\delta_{A}^{B}$, and $\epsilon_{a c} \epsilon^{b c}=$ $-\delta_{a}^{c}$. It is useful to introduce a vector field variable for the gradient of $r$ :

$$
w_{A} \equiv r^{-1} r_{\mid A} .
$$

Then, any covariant derivative on the spacetime can be written in terms of the covariant derivatives on $M^{2}$ and $S^{2}$, plus some terms due to the warp factor $r^{2}$, which can be written in terms of $w_{A}$.

Metric linear perturbations of a spherically-symmetric background can be decomposed in scalar, vector and tensor spherical harmonics [40, 41]. The scalar spherical harmonics $Y^{\ell m}$ are eigenfunctions of the covariant Laplacian on the sphere:

$$
\gamma^{a b} Y_{: a b}^{\ell m}=-l(l+1) Y^{\ell m} .
$$

A basis of vector spherical harmonics (defined for $l \geq 1$ ) is

$$
Y_{a}^{\ell m} \equiv Y_{: a}^{\ell m}, \quad S_{a}^{\ell m} \equiv \epsilon_{a}^{b} Y_{b}^{\ell m},
$$

where the $Y_{a}^{\ell m}$ 's have polar parity (they transform as $(-1)^{l}$, like the scalar harmonics, under parity transformations, and are also called even-parity type) and the $S_{a}^{\ell m}$ 's have axial parity (they transform as $(-1)^{l+1}$ under parity transformations, and are also called odd-parity type). A basis of tensor spherical harmonics (defined for $l \geq 2)$ is

$$
\begin{gathered}
Y_{a b}^{\ell m} \equiv Y^{\ell m} \gamma_{a b}, \quad Z_{a b}^{\ell m} \equiv Y_{: a b}^{\ell m}+\frac{l(l+1)}{2} Y^{\ell m} \gamma_{a b}, \\
S_{a b}^{\ell m} \equiv S_{(a: b)}^{\ell m},
\end{gathered}
$$

where the $Y_{a b}^{\ell m}, Z_{a b}^{\ell m}$ have polar parity and the $S_{a b}^{\ell m}$ have axial parity.

We then split the metric perturbations $h_{\alpha \beta}$ into polar and axial perturbations, $h_{\alpha \beta}=h_{\alpha \beta}^{\mathrm{a}}+h_{\alpha \beta}^{\mathrm{p}}$, and these can be expanded in the basis of tensor harmonics as

$$
h_{\alpha \beta}^{\mathrm{a}}=\sum_{\ell, m} h_{\alpha \beta}^{\mathrm{a}, \ell m}, \quad h_{\alpha \beta}^{\mathrm{p}}=\sum_{\ell, m} h_{\alpha \beta}^{\mathrm{p}, \ell m},
$$

where

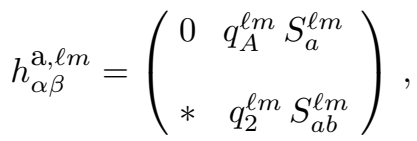

$$
\begin{aligned}
& h_{\alpha \beta}^{\mathrm{p}, \ell m}=\left(\begin{array}{cc}
h_{A B}^{\ell m} Y^{\ell m} & h_{A}^{\ell m} Y_{a}^{\ell m} \\
* & r^{2}\left(K^{\ell m} Y_{a b}^{\ell m}+G^{\ell m} Z_{a b}^{\ell m}\right)
\end{array}\right),
\end{aligned}
$$

and where we use asterisks to denote the symmetry of these tensors. All the perturbations, $h_{A B}^{\ell m}$ (scalar), $h_{A}^{\ell m}$ and $q_{A}^{\ell m}$ (vector), and $K^{\ell m}, G^{\ell m}$, and $q_{2}^{\ell m}$ (tensor), depend only on the coordinates of the 2-manifold $M^{2}$.

The components of the energy-momentum tensor of a point-like object are given by

$$
T^{\alpha \beta}=m \int \frac{d \tau}{\sqrt{-g}} u^{\alpha} u^{\beta} \delta^{4}[x-z(\tau)],
$$


where $m$ is the mass, $\tau$ denotes proper time, $z(\tau)$ is the trajectory of the object, $g$ denotes the metric determinant, and $\delta^{4}$ is the four-dimensional Dirac density $\left(\int d^{4} x \sqrt{-g} \delta^{4}(x)=1\right)$. We choose to decompose in harmonics the contravariant components $T^{\alpha \beta}$ [the decomposition for the covariant ones follows immediately]. In this way, the polar components can be described in terms of the following quantities

$$
\begin{gathered}
Q_{\ell m}^{A B}=8 \pi \int_{S^{2}} d \Omega T^{A B} \bar{Y}^{\ell m}, \\
Q_{\ell m}^{A}=\frac{16 \pi r^{2}}{\ell(\ell+1)} \int_{S^{2}} d \Omega T^{A a} \bar{Y}_{a}^{\ell m}, \\
Q_{\ell m}^{Y}=8 \pi r^{2} \int_{S^{2}} d \Omega T^{a b} \bar{Y}_{a b}^{\ell m} \\
Q_{\ell m}^{Z}=32 \pi r^{4} \frac{(\ell-2) !}{(\ell+2) !} \int_{S^{2}}^{d \Omega T^{a b}} \bar{Z}_{a b}^{\ell m},
\end{gathered}
$$

where the bar denotes complex conjugation. The axial components can be described in terms of the quantities

$$
\begin{gathered}
P_{\ell m}^{A}=\frac{16 \pi r^{2}}{\ell(\ell+1)} \int_{S^{2}} d \Omega T^{A a} \bar{S}_{a}^{\ell m}, \\
P_{\ell m}=16 \pi r^{4} \frac{(\ell-2) !}{(\ell+2) !} \int_{S^{2}} d \Omega T^{a b} \bar{S}_{a b}^{\ell m},
\end{gathered}
$$

To simplify the equations we choose to work in the Regge-Wheeler gauge:

$$
h_{A}^{\ell m}=G^{\ell m}=0, \quad q_{2}^{\ell m}=0
$$

but a fully covariant and gauge-invariant approach can be found in 31].

The perturbative equations can be decoupled by introducing certain combinations of the metric perturbations, which are gauge-invariant. For the axial modes, it is common to use the Regge-Wheeler master function

$$
\Psi_{\ell m}^{\mathrm{RW}}=-\frac{1}{r} w^{A} q_{A}^{\ell m},
$$

however, in this paper we will use the master function introduced by Cunningham, Price and Moncrief [21], following the definition used in 31,42$]$ :

$$
\Psi_{\ell m}^{\mathrm{CPM}}=\frac{2 r}{(\ell+2)(\ell-1)} \epsilon^{A B}\left(q_{B \mid A}^{\ell m}-\frac{2}{r} w_{A} q_{B}^{\ell m}\right) .
$$

One reason for using this function is to have the formulas for the energy and angular momentum radiated to coincide with the ones for the polar modes (see below). In this respect, the formulation for axial modes is on an equal footing with the one for polar modes. For the polar modes, we use the well-known Zerilli-Moncrief master function:

$$
\Psi_{\ell m}^{\mathrm{ZM}}=\frac{2 r}{\ell(\ell+1)}\left[K^{\ell m}+\frac{1}{\Lambda}\left(h_{A B}^{\ell m} w^{A} w^{b}-r w^{A} K_{\mid A}^{\ell m}\right)\right],
$$

where $\Lambda=(\ell+2)(\ell-1) / 2+3 M / r$. The key point in introducing these quantities is the fact that the Einstein perturbative equations can be decoupled for them, and the remaining perturbative variables can be recovered from them. As it is well know, the equations for $\Psi_{\ell m}^{\mathrm{CPM}}$ (or $\left.\Psi_{\ell m}^{\mathrm{RW}}\right)$ and $\Psi_{\ell m}^{\mathrm{ZM}}$ are wave-type equations of the form:

$$
\left[-\partial_{t}^{2}+\partial_{r_{*}}^{2}-V_{\ell}^{\mathrm{RW} / \mathrm{zM}}(r)\right] \Psi_{\ell m}^{\mathrm{CPM} / \mathrm{ZM}}=f \mathcal{S}_{\ell m}^{\mathrm{CPM} / \mathrm{ZM}},
$$

where $r_{*}$ is the so-called tortoise coordinate $\left(r_{*}=r+\right.$ $2 M \ln (r /(2 M)-1))$. The potential for the axial modes is the Regge-Wheeler potential

$$
V_{\ell}^{\mathrm{RW}}(r)=\frac{f}{r^{2}}\left(\ell(\ell+1)-\frac{6 M}{r}\right),
$$

and the one for polar modes is the Zerilli potential

$$
V_{\ell}^{\mathrm{ZM}}(r)=\frac{f}{r^{2} \Lambda^{2}}\left[2 \lambda_{\ell}^{2}\left(1+\lambda_{\ell}+\frac{3 M}{r}\right)+18 \frac{M^{2}}{r^{2}}\left(\lambda_{\ell}+\frac{M}{r}\right)\right],
$$

where $\lambda_{\ell}=(\ell+2)(\ell-1) / 2$. The source term for axial modes is given by

$$
\mathcal{S}_{\ell m}^{\mathrm{CPM}}=\frac{2 r}{\ell(\ell+1)} \epsilon^{A B} P_{A \mid B}^{\ell m}
$$

and for polar modes by

$$
\begin{aligned}
\mathcal{S}_{\ell m}^{\mathrm{ZM}} & =\frac{2}{\Lambda} w_{A} Q_{\ell m}^{A}-\frac{1}{r} Q_{\ell m}^{Z}-\frac{r^{2}}{\left(1+\lambda_{\ell}\right) \Lambda}\left\{w^{C} \mathrm{~g}_{A B} Q_{\ell m \mid C}^{A B}-\frac{6 M}{r^{2} \Lambda} w_{A} w_{B} Q_{\ell m}^{A B}-\frac{f}{r} Q_{\ell m}^{Y}\right. \\
& \left.-\frac{1}{r \Lambda}\left[\lambda_{\ell}\left(\lambda_{\ell}-1\right)+3\left(2 \lambda_{\ell}-3\right) \frac{M}{r}+21 \frac{M^{2}}{r^{2}}\right] \mathrm{g}_{A B} Q_{\ell m}^{A B}\right\} .
\end{aligned}
$$

When we restrict ourselves to the case of a point particle, by introducing the energy-momentum tensor (12) into 
the previous expressions, we find that the source term for both polar and axial modes has the following (singular) structure:

$$
\mathcal{S}(t, r)=G(t, r) \delta\left[r-r_{p}(t)\right]+F(t, r) \delta^{\prime}\left[r-r_{p}(t)\right],
$$

where $\delta$ is the one-dimensional Dirac delta distribution and $\delta^{\prime}$ its first derivative. The function $r_{p}(t)$ describes the radial motion of the particle in terms of the coordinate time $t$. In the polar case, the explicit expressions for the functions $F(t, r)$ and $G(t, r)$ can be found, for instance, in 32,38 . They are given by

$$
\begin{aligned}
G_{\ell m}^{\mathrm{ZM}}(t, r) & =a_{\ell}(r) \bar{Y}^{\ell m}(t)+b_{\ell}(r) \bar{Y}_{\varphi}^{\ell m}(t) \\
& +c_{\ell}(r) \bar{Y}_{\varphi \varphi}^{\ell m}(t)+d_{\ell}(r) \bar{Z}_{\varphi \varphi}^{\ell m}(t)
\end{aligned}
$$

$$
F_{\ell m}^{\mathrm{ZM}}(t, r)=A_{\ell}(r) \bar{Y}^{\ell m}(t)
$$

where the $t$-dependence in the right-hand side has to be understood as: $(t)=\left(\theta=\theta_{p}=\pi / 2, \varphi=\varphi_{p}(t)\right)$. The different functions of $r$ are given by:

$$
\begin{aligned}
& a_{\ell}(r)=\frac{8 \pi m}{1+\lambda_{\ell}} \frac{f^{2}}{r \Lambda^{2}}\left\{\frac{6 M}{r} E_{p}-\frac{\Lambda}{E_{p}}\left[1+\lambda_{\ell}-\frac{3 M}{r}+\frac{L_{p}^{2}}{r^{2}}\left(\lambda_{\ell}+3-\frac{7 M}{r}\right)\right]\right\} \\
& b_{\ell}(r)=\frac{16 \pi m}{1+\lambda_{\ell}} \frac{L_{p}}{E_{p}} \frac{f^{2}}{r^{2} \Lambda} u^{r}, \\
& c_{\ell}(r)=\frac{8 \pi m}{1+\lambda_{\ell}} \frac{L_{p}^{2}}{E_{p}} \frac{f^{3}}{r^{3} \Lambda} \\
& d_{\ell}(r)=-32 \pi m \frac{(\ell-2) !}{(\ell+2) !} \frac{L_{p}^{2}}{E_{p}} \frac{f^{2}}{r^{3}}, \\
& A_{\ell}(r)=\frac{8 \pi m}{1+\lambda_{\ell}} \frac{f^{3}}{\Lambda} \frac{1}{E_{p}}\left(1+\frac{L_{p}^{2}}{r^{2}}\right) \\
& G_{\ell m}^{\mathrm{CPM}}(t, r)=u_{\ell}(r) \bar{S}_{\varphi}^{\ell m}(t)+v_{\ell}(r) \bar{S}_{\varphi \varphi}^{\ell m} \\
& F_{\ell m}^{\mathrm{CPM}}(t, r)=B_{\ell}(r) \bar{S}_{\varphi}^{\ell m}(t)
\end{aligned}
$$

where $E_{p}$ is the particle energy per unit mass, $L_{p}$ is the orbital angular momentum, and $u^{r}$ is the radial compo-

where

$$
\begin{gathered}
u_{\ell}(r)=32 \pi m \frac{(\ell-2) !}{(\ell+2) !} \frac{f^{2}}{r^{2}} \frac{L_{p}}{E_{p}^{2}}\left[\left(1-\frac{5 M}{r}\right)\left(1+\frac{L_{p}^{2}}{r^{2}}\right)+f \frac{L_{p}^{2}}{r^{2}}-2 E_{p}^{2}\right], \\
v_{\ell}(r)=32 \pi m \frac{(\ell-2) !}{(\ell+2) !} \frac{f^{2}}{r^{3}} \frac{L_{p}^{2}}{E_{p}^{2}} u^{r}, \quad \text { (39) } \begin{array}{l}
\text { It is worth pointing out that for circular motion, there is } \\
\text { only contribution from the vector harmonics. }
\end{array} \\
\begin{array}{l}
\text { The singular structure of the source terms in the mas- } \\
\text { ter equations, for both polar and axial modes, shown } \\
\text { in equation (28) implies the existence of discontinuities } \\
\text { in the master function at the particle's location. Out- }
\end{array}
\end{gathered}
$$


side the location of the particle the solution is smooth (assuming it was initially). One can then divide the onedimensional spatial domain into two different and disjoint regions (which will change in time as the particle moves): The region to the left of the particle $\left(r<r_{p}(t)\right)$, and the region to the right of the particle $\left(r>r_{p}(t)\right)$. The master functions, on that regions, satisfy an homogeneous wavelike equation [Equation (53) without sources]. Then, we can think of the solution of the full equation as being composed of the solutions of the homogeneous equations to the left and to the right of the particle, and relations between them at the particle location. Obviously these relations consist of imposing the discontinuities that the singular source terms dictate. In other words, we can write the solution of the full equation as

$$
\Psi(t, r)=\Psi^{-}(t, r) \theta\left(r_{p}(t)-r\right)+\Psi^{+}(t, r) \theta\left(r-r_{p}(t)\right),
$$

where $\theta(r)$ is the Heaviside step function, and $\Psi^{+}(t, r)$ and $\Psi^{-}(t, r)$ are solutions of the homogeneous equation to the right and to the left of the particle respectively. Due to the existence of the particle we will have that

$$
\Psi^{-}\left(t, r_{p}\right) \neq \Psi^{+}\left(t, r_{p}\right), \quad\left(\partial_{r} \Psi^{-}\right)\left(t, r_{p}\right) \neq\left(\partial_{r} \Psi^{+}\right)\left(t, r_{p}\right) .
$$

By introducing (41) into the full equation we can derive the equation that govern the jumps in the master functions. The result is:

$$
\begin{gathered}
\left(f^{2}(t)-\dot{r}_{p}^{2}(t)\right)\left[\partial_{r} \Psi\right]-2 \dot{r}_{p}(t) \partial_{t}[\Psi] \\
-\left(\ddot{r}_{p}(t)+f(t) f^{\prime}(t)\right)[\Psi]=\mathcal{G}\left(t, r_{p}(t)\right), \\
\left(f^{2}(t)+\dot{r}_{p}^{2}(t)\right)[\Psi]=\mathcal{F}\left(t, r_{p}(t)\right),
\end{gathered}
$$

where $f(t) \equiv f\left(r_{p}(t)\right)$, and $[\Psi]$ and $\left[\partial_{r} \Psi\right]$ are the master function discontinuities at the particle location

$$
\begin{gathered}
{[\Psi]=\lim _{r \rightarrow r_{p}(t)} \Psi^{+}(t, r)-\lim _{r \rightarrow r_{p}(t)} \Psi^{-}(t, r),} \\
{\left[\partial_{r} \Psi\right]=\lim _{r \rightarrow r_{p}(t)} \partial_{r} \Psi^{+}(t, r)-\lim _{r \rightarrow r_{p}(t)} \partial_{r} \Psi^{-}(t, r),}
\end{gathered}
$$

where $\mathcal{F}$ and $\mathcal{G}$ are functions of $t$ and the particle location $r_{p}(t)$ that one obtains after applying the following properties of the Dirac delta distribution

$$
\begin{aligned}
A(t, r) \delta\left(r-r_{p}(t)\right) & =A\left(t, r_{p}(t)\right) \delta\left(r-r_{p}(t)\right) \\
A(t, r) \delta^{\prime}\left(r-r_{p}(t)\right) & =-\left(\partial_{r} A\right)\left(t, r_{p}(t)\right) \delta\left(r-r_{p}(t)\right) \\
& +A\left(t, r_{p}(t)\right) \delta^{\prime}\left(r-r_{p}(t)\right)
\end{aligned}
$$

to the original source terms (28). The equations for the discontinuities [Equations (43) and (44)] contain the particle radial position $r_{p}(t)$, and its first and second time derivatives $\left(\dot{r}_{p}(t), \ddot{r}_{p}(t)\right)$. The first two, $r_{p}(t)$ and $\dot{r}_{p}(t)$, are obtained via numerical integration of the geodesics ODEs shown in Appendix $\AA$ and the third one, $\ddot{r}_{p}(t)$, can be found directly from the geodesic equations:

$$
\begin{aligned}
\ddot{r}_{p}(t) & =\frac{f^{2}(t)}{r_{p}^{2}(t)} \frac{L_{p}^{2}}{E_{p}^{2}}\left(\frac{f(t)}{r_{p}(t)}-\frac{3 f^{\prime}(t)}{2}\right) \\
& +f(t) f^{\prime}(t)\left(1-\frac{3 f^{2}(t)}{2 E_{p}^{2}}\right) .
\end{aligned}
$$

We finish this section by giving the expressions of the averaged energy at angular momentum luminosities at infinity (as obtained from the Isaacson's averaged energymomentum tensor for gravitational waves [43, 44]), which also hold at the horizon, in terms of the axial and polar master functions:

$$
\begin{gathered}
\dot{E}=\frac{1}{64 \pi} \sum_{\ell \geq 2, m} \frac{(\ell+2) !}{(\ell-2) !}\left(\left|\dot{\Psi}_{\ell m}^{\mathrm{CPM}}\right|^{2}+\left|\dot{\Psi}_{\ell m}^{\mathrm{ZM}}\right|^{2}\right) \\
\dot{L}=\frac{1}{64 \pi} \sum_{\ell \geq 2, m} i m \frac{(\ell+2) !}{(\ell-2) !}\left(\bar{\Psi}_{\ell m}^{\mathrm{CPM}} \dot{\Psi}_{\ell m}^{\mathrm{CPM}}+\bar{\Psi}_{\ell m}^{\mathrm{ZM}} \dot{\Psi}_{\ell m}^{\mathrm{ZM}}\right) .
\end{gathered}
$$

Finally, the metric waveforms are given by

$$
h_{+}-i h_{\times}=\frac{1}{2 r} \sum_{\ell \geq 2, m} \sqrt{\frac{(\ell+2) !}{(\ell-2) !}}\left\{\Psi_{\ell m}^{\mathrm{ZM}}+i \Psi_{\ell m}^{\mathrm{CPM}}\right\}_{-2} Y^{\ell m},
$$

where ${ }_{-2} Y^{\ell m}$ are the spherical harmonics of spin weight -2 (see, e.g. [45]).

\section{THE NUMERICAL FRAMEWORK}

In this section we introduce the basics on the numerical framework that we use to solve numerically the perturbative equations described above. This task involves a number of choices that determine the particular features of our numerical method. To be specific, we want to solve our equations in the time domain, that is, we want to develop an algorithm that evolves the initial data from an initial state to a final time where we are interested in knowing the solution. This implies a discretization of our equations both in space and time. We choose to discretize in space by using a Galerkin-type FE procedure, and in time by using Finite Differences techniques. In what follows we describe the details of these ingredients of our numerical calculations.

\section{A. The Mathematical Formulation of the Problem}

The model PDE problem that we are interesting in solving has the following form:

$$
\mathcal{L}[\Psi] \equiv\left(-\partial_{t}^{2}+\partial_{x}^{2}-V(x)\right) \Psi(t, x)-\mathcal{S}(t, x)=0,
$$




$$
\begin{gathered}
\Psi\left(t_{o}, x\right)=\psi_{o}(x), \quad\left(\partial_{t} \Psi\right)\left(t_{o}, x\right)=\dot{\psi}_{o}(x), \\
{\left[\left(\partial_{t}-\partial_{x}\right) \Psi\right]\left(t, x_{H}\right)=0, \quad\left[\left(\partial_{t}+\partial_{x}\right) \Psi\right]\left(t, x_{I}\right)=0,}
\end{gathered}
$$

where

$$
t \in\left[t_{o}, t_{f}\right], \text { and } x \in \Omega=\left[x_{H}, x_{I}\right]
$$

Expression (53) presents the structure of the equations we need to solve, namely a wave equation in a potential $V$ and with a source $\mathcal{S}$. Here, $x$ corresponds to the tortoise coordinate $r_{*}$ in the master equations, and for the sake of simplicity of the notation we will use it in most of the rest of the paper. The form of $\mathcal{S}(t, x)$ is assumed to be known, as it is in our case. Equation (54) represents the initial conditions for the time evolution, we need the initial value of $\Psi$ and its time derivative to solve a second-order hyperbolic problem. The boundary conditions, given in (55), are simple one-dimensional outgoing conditions (also known as Sommerfeld boundary conditions) at both ends of the spatial interval where the equation is considered, specified in (56). This boundary condition is an exact outgoing boundary condition only at infinity, provided the potential and the source have a fall-off of the type of the potentials and sources that we are considering in our physical problem, otherwise it is just an approximate outgoing boundary condition whose accuracy depends on how far $x_{I}$ is located. A similar argument also holds for the boundary condition at $x_{H}$, the accuracy of the boundary condition that we use depends on how far towards $-\infty$ we locate $x_{H}$. There is a well-known difference between $x \rightarrow \infty$ and $x \rightarrow-\infty$ which is due to the asymptotic behaviour of the potentials. When we approach $\rightarrow \infty$ the potentials behave like const. $\times x^{-2}$, whereas when we approach $\rightarrow-\infty$ the potentials behave like const. $\times \exp \{-x /(2 M)\}$, therefore our approximate boundary conditions should work much better at $x_{H}$ than at $x_{I}$ since our equations resemble the wave equation better around $x_{H}$. One could also get better boundary conditions by using the methods suggested in references [46, 47], where higher-order derivative boundary conditions are proposed, or by using the methods proposed in [48, 49, 50, 51, 52] where exact radiative boundary conditions are studied.

\section{B. The Finite Element Discretization}

In this section we describe, in a simplified way, the main ingredients of the FEM that are relevant for our calculations. Detailed exposions can be found in [53, 54, $55,56]$.

The way one discretizes in space in a FE framework can be summarized in the following three steps: (i) Domain discretization. The division of the spatial domain
$\Omega$ into a collection of disjoint subdomains $\left\{\Omega_{k}\right\}_{i=1 \ldots N}$, the elements. (ii) The FE functional space. At every element, $\Omega_{k}$, we introduce a finite-dimensional functional space, $\mathcal{F}_{k}$, that we use to expand our fields locally at $\Omega_{k}$. Typically, these functional spaces are made out of polynomials. (iii) Weak formulation of the equation. This consists in converting the differential form of the equations into an integral form that involves the boundary conditions of the von Neumann type. (iv) Equation discretization. In a Garlekin-type of FE formulation, the discretized equations are obtain throught the imposition of the vanishing of all the residuals, $\mathcal{E}_{A} \equiv \int_{\Omega} d x n_{i} \mathcal{L}[\Psi]$, the components of our equation with respect a basis of nodal functions $\left\{n_{i}(x)\right\}$ built out of the spaces $\mathcal{F}_{k}$ (see below).

\section{Domain Discretization and the FE functional spaces}

Points (i) and (ii) have to be treated jointly, because the structure of the elements and the structure of the FE functional spaces are not completely independent.

Since we are dealing with a one-dimensional problem, the first step, the subdivision of the domain, is quite simple, we just divide the interval $\left[x_{H}, x_{I}\right]$ into subintervals (see Figure 11): $\Omega_{1}=\left[x_{H}, x_{1}\right), \Omega_{2}=\left[x_{1}, x_{2}\right), \ldots$, $\Omega_{N}=\left[x_{N-1}, x_{I}\right]$. This equivalent to locate $N+1$ nodes in an ordered way: $x_{0} \equiv x_{H}<x_{1}<\cdots<x_{N-1}<x_{N} \equiv$ $x_{I}$. We denote the size (length) of the element $\Omega_{k}$ by $d_{k}=x_{k+1}-x_{k}$.

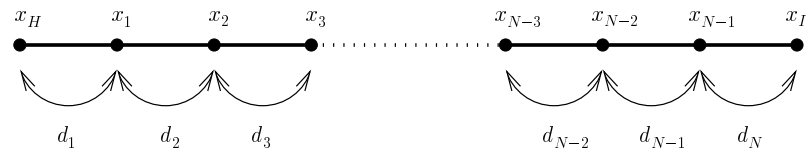

FIG. 1: One-dimensional Mesh.

The second step is very important in the sense that the accuracy and convergence properties of the FE scheme depend on the choice of the FE functional space. In this paper, we restrict ourselves to linear elements. For sufficiently regular meshes, linear elements lead to secondorder convergence in the $L^{2}$ norm. On the other hand, the functional spaces $\mathcal{F}_{k}$ for linear elements are twodimensional and can be span by the following two functions (see Figure 2):

$$
\begin{gathered}
M_{i}(x)= \begin{cases}\frac{x-x_{i}}{d_{i}} & \text { if } x \in\left(x_{i}, x_{i+1}\right), \\
0 & \text { otherwise }\end{cases} \\
N_{i}(x)= \begin{cases}\frac{x_{i+1}-x}{d_{i}} & \text { if } x \in\left(x_{i}, x_{i+1}\right), \\
0 & \text { otherwise }\end{cases}
\end{gathered}
$$

which are usually called linear interpolation functions. However, to build a FE approximation of the solution of 
our PDEs it is more convenient to use the nodal functions, $n_{i}(x)$ :

$$
n_{H}(x)=N_{H}(x), \quad n_{I}(x)=M_{N-1}(x) .
$$

and for $i=1, \ldots, N-1$ :

$$
n_{i}(x)= \begin{cases}M_{i-1}(x) & \text { if } x \in\left(x_{i-1}, x_{i}\right) \\ N_{i}(x) & \text { if } x \in\left(x_{i}, x_{i+1}\right) \\ 0 & \text { otherwise }\end{cases}
$$

Their are called nodal functions because of the following property (see Figure 3):

$$
n_{i}\left(x_{j}\right)=\delta_{i j}
$$

that is, they vanish at all nodes excepting at the one they are associated with, where they take the unity value.

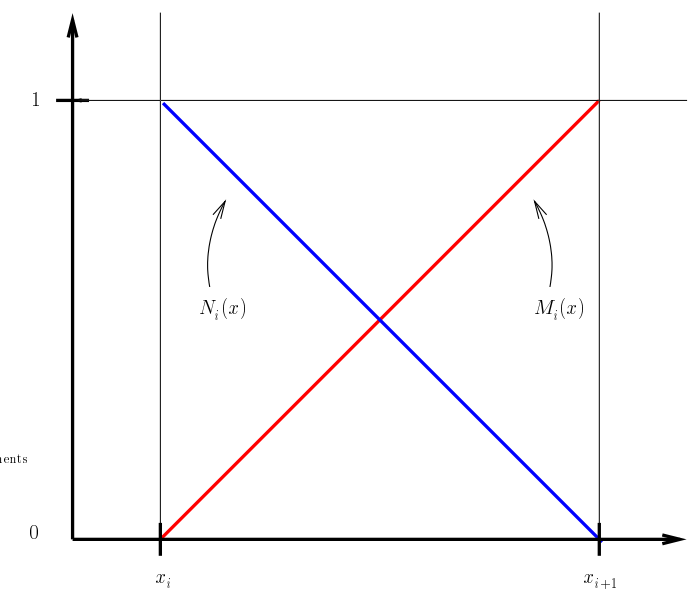

FIG. 2: Linear interpolation functions $M_{i}(x)$ and $N_{i}(x)$.

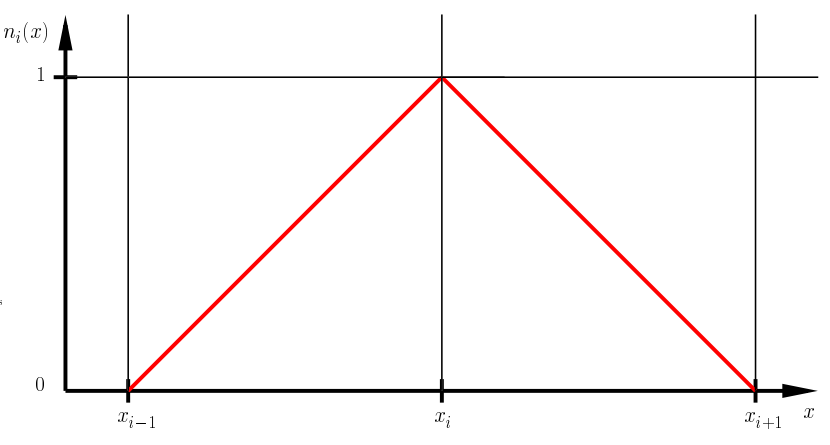

FIG. 3: Nodal functions $n_{i}(x)$.

\section{The weak form of the equations}

The next step is to formulate our problem in what is called the weak form of the equation, which is an integral form. To that end, let us consider an arbitrary test function $\phi$ on $\left[t_{o}, t_{f}\right] \times\left[x_{H}, x_{I}\right]$ and multiply equation (53) by it. Then, we integrate over $\left[x_{H}, x_{I}\right]$ and apply integration by parts to the term with second spatial derivatives. This produces a boundary term with first spatial derivatives that can be converted into first time derivatives by using our boundary conditions (55). The result is:

$$
\mathcal{E}[\phi, \Psi]=-\int_{x_{H}}^{x_{I}} d x\left[\phi \partial_{t}^{2} \Psi+\left(\partial_{x} \phi\right)\left(\partial_{x} \Psi\right)+V(x) \phi \Psi\right]+\left.\phi \partial_{t} \Psi\right|_{x_{I}}+\left.\phi \partial_{t} \Psi\right|_{x_{H}}-\int_{x_{H}}^{x_{I}} d x \phi \mathcal{S}=0
$$

This is the weak form of our equation, which has the remarkable property of including the boundary conditions of the problem. In the case we were dealing with Dirichlet boundary conditions, usually called essential boundary conditions in the FEM language, they would have not been incorporated in the weak formulation of the equation. Instead, they are used to eliminate unknowns by providing values for them.

\section{The FE discretization of the equation}

To find the FE discretization of our equations we need to introduce the FE approximation to the solution of our problem. This approximation consists in an expansion in the nodal functions

$$
\Psi_{h}(t, x)=\sum_{i=0}^{N} \psi_{i}(t) n_{i}(x)
$$

where the time-dependent functions $\psi_{i}(t)$ are going to be the unknowns of our problem. Then, the FE discretization of the equations of our problem consists in imposing the vanishing of the residuals:

$$
\mathcal{E}_{i} \equiv \mathcal{E}\left[n_{i}, \Psi_{h}\right]=0, \quad i=0, \ldots, N
$$


This leads to one equation for node ${ }^{1}$, or equivalently, we have $N+1$ equations for our $N+1$ unknowns $\left\{\psi_{i}\right\}$. By introducing the FE approximation (63) into the weak form of our equation (62) and imposing (64), we get a system of coupled ODEs for the unknowns. We can write it in a matrix form as follows:

$$
\mathbb{M} \cdot \ddot{\Psi}+\mathbb{G} \cdot \dot{\Psi}+\mathbb{K} \cdot \Psi=\boldsymbol{F},
$$

where $\mathbb{M}, \mathbb{G}$, and $\mathbb{K}$ are $(N+1) \times(N+1)$ matrices, and $\boldsymbol{\Psi}$ and $\boldsymbol{F}$ are vectors with $N+1$ components, and $\ddot{\boldsymbol{\Psi}}$ and $\dot{\boldsymbol{\Psi}}$ are the time derivatives of $\boldsymbol{\Psi}$. The names and meaning of these objects can be thought to be inspired in the analogy of the system of equations (65) with the system of equations for a coupled system of oscillators. The matrix $\mathbb{M}$ is usually called the mass matrix and its components are:

$$
\mathbb{M}_{i j}=\int_{x_{H}}^{x_{I}} d x n_{i}(x) n_{j}(x),
$$

and hence it is a symmetric and positive definite matrix. The matrix $\mathbb{G}$ is usually called the damping matrix and has components

$$
\mathbb{G}_{i j}=n_{i}\left(x_{H}\right) n_{j}\left(x_{H}\right)+n_{i}\left(x_{I}\right) n_{j}\left(x_{I}\right),
$$

which is symmetric and only has contributions from the boundaries, which means that our system only dissipates (or absorbs, if they sign of these two terms would have been negative, corresponding to ingoing boundary conditions) energy ${ }^{2}$ through the boundaries and this reflects the fact that we are using outgoing boundary conditions. The matrix $\mathbb{K}$ is usually called the stiffness matrix. Its components are given by

$$
\mathbb{K}_{i j}=\int_{x_{H}}^{x_{I}} d x\left[n_{i}^{\prime}(x) n_{j}^{\prime}(x)+V(x) n_{i}(x) n_{j}(x)\right],
$$

therefore, it is also symmetric. Finally, $\boldsymbol{\Psi}=\left(\psi_{i}(t)\right)$ and $\boldsymbol{F}$ is usually called the force vector and its components are given by

$$
\boldsymbol{F}_{i}(t)=-\int_{x_{H}}^{x_{I}} d x \mathcal{S}(t, x) n_{i}(x) .
$$

In our particular case, we can compute most of the components of the matrices and vectors that determine

${ }^{1}$ In the case of problems involving essential boundary conditions we would get as many equations as unknows remain after imposing these boundary conditions.

${ }^{2}$ We can define an energy for our system by:

$$
\mathrm{E}[\boldsymbol{\Psi}, \dot{\Psi}]=\frac{1}{2}\left(\dot{\boldsymbol{\Psi}}^{\mathrm{T}} \cdot \mathbb{M} \cdot \dot{\Psi}+\boldsymbol{\Psi}^{\mathrm{T}} \cdot \mathbb{K} \cdot \boldsymbol{\Psi}\right)
$$

This energy would be preserved by the evolution when $\mathbb{G}=\boldsymbol{F}=$ 0 . our system of ODEs (65) analytically. The expressions for the components of mass matrix are:

$$
\mathbb{M}_{i j}=\frac{1}{6}\left[d_{i-1} \delta_{i-1 j}+2\left(d_{i-1}+d_{i}\right) \delta_{i j}+d_{i} \delta_{i+1 j}\right]
$$

for $i, j,=1, \ldots, N-1$, and the components related to the boundaries are: $\mathbb{M}_{H H}=d_{H} / 3, \mathbb{M}_{H 1}=d_{H} / 6, \mathbb{M}_{N-1 I}=$ $d_{N-1} / 6$, and $\mathbb{M}_{I I}=d_{N-1} / 3$. The components of the damping matrix are simply given by

$$
\mathbb{G}_{i j}=\delta_{i H} \delta_{j H}+\delta_{i I} \delta_{j I},
$$

The first term of the components of the stiffness matrix is given by

$$
\mathbb{K}_{i j}=-\frac{1}{d_{i-1}} \delta_{i-1 j}+\left(\frac{1}{d_{i-1}}+\frac{1}{d_{i}}\right) \delta_{i j}-\frac{1}{d_{i-1}} \delta_{i+1 j}
$$

for $i, j,=1, \ldots, N-1$, and the components related to the boundaries are: $\mathbb{K}_{H H}=1 / d_{H}, \mathbb{K}_{H 1}=-1 / d_{H}$, $\mathbb{K}_{N-1 I}=-1 / d_{N-1}$, and $\mathbb{K}_{I I}=-1 / d_{N-1}$. The second term in the components of the stiffness matrix involves the potential, and therefore it has to be computed numerically. To that end, we use Gauss-Legendre quadratures (see Appendix B).

We can compute the components of the force vector $\boldsymbol{F}$ by using the form of the source term $\mathcal{S}$, which is given in equation (28). Using the properties of the Dirac delta distribution, we find that the structure of $\mathcal{S}$ implies the following structure for the components of the force vector:

$$
\begin{aligned}
\boldsymbol{F}_{i}(t) & =\left\{\nu\left(r_{p}(t)\right)\left[\left(\partial_{r} F\right)\left(t, r_{p}(t)\right)-G\left(t, r_{p}(t)\right)\right]\right. \\
& \left.+\nu^{\prime}\left(r_{p}(t)\right) F\left(t, r_{p}(t)\right)\right\} n_{i}\left(x_{p}(t)\right) \\
& +\nu^{2}\left(r_{p}(t)\right) F\left(t, r_{p}(t)\right) n_{i}^{\prime}\left(x_{p}(t)\right)
\end{aligned}
$$

where

$$
\nu(r)=\frac{d x(r)}{d r}=\frac{1}{f}, \quad \nu^{\prime}(r)=\frac{d \nu(r)}{d r}=-\frac{2 M}{r^{2} f^{2}},
$$

and $x_{p}(t)$ is just the radial motion in terms of the tortoise coordinate. This completes the FE discretization of our problem.

\section{Evolution Algorithms}

The next step is to solve the system of ODEs given in equation (65), which is coupled to the ODEs corresponding to the motion of the point-like object [equations A4 A5 in Appendix A. The numerical algorithms we use derive from the average acceleration method, which is based on the assumption that over a small time interval any nodal acceleration can be considered to be a linear function of time. Then, for a time interval $\left(t_{o}, t_{o}+\Delta t\right)$, we write

$$
\ddot{\mathbf{\Psi}}(t)=\ddot{\mathbf{\Psi}}\left(t_{o}\right)\left(1-\frac{t}{\Delta t}\right)+\ddot{\mathbf{\Psi}}\left(t_{o}+\Delta t\right) \frac{t}{\Delta t} .
$$


Integrating in time this equation twice and evaluating at $t=t_{1}=t_{o}+\Delta t$ we get

$$
\begin{gathered}
\dot{\boldsymbol{\Psi}}\left(t_{1}\right)=\dot{\boldsymbol{\Psi}}\left(t_{o}\right)+\frac{1}{2}\left[\ddot{\mathbf{\Psi}}\left(t_{o}\right)+\ddot{\mathbf{\Psi}}\left(t_{1}\right)\right] \Delta t, \quad(76) \\
\mathbf{\Psi}\left(t_{1}\right)=\mathbf{\Psi}\left(t_{o}\right)+\dot{\mathbf{\Psi}}\left(t_{o}\right) \Delta t+\frac{1}{6}\left[2 \ddot{\mathbf{\Psi}}\left(t_{o}\right)+\ddot{\mathbf{\Psi}}\left(t_{1}\right)\right](\Delta t)^{2} .
\end{gathered}
$$

This algorithm that one derives from these expressions is conditionally stable. Newmark [57] introduced a generalization of the equations (76) and (77) in the following way

$$
\begin{aligned}
\dot{\Psi}\left(t_{1}\right)= & \dot{\Psi}\left(t_{o}\right)+\left[(1-\gamma) \ddot{\mathbf{\Psi}}\left(t_{o}\right)+\gamma \ddot{\mathbf{\Psi}}\left(t_{1}\right)\right] \Delta t, \\
\mathbf{\Psi}\left(t_{1}\right) & =\boldsymbol{\Psi}\left(t_{o}\right)+\dot{\mathbf{\Psi}}\left(t_{o}\right) \Delta t \\
& +\frac{1}{2}\left[(1-2 \beta) \ddot{\mathbf{\Psi}}\left(t_{o}\right)+2 \beta \ddot{\mathbf{\Psi}}\left(t_{1}\right)\right](\Delta t)^{2},
\end{aligned}
$$

where $(\gamma, \beta)$ are parameters that have to be chosen for accuracy and stability. The Newmark method is unconditionally stable for the following range of the parameters $(\gamma, \beta)$ :

$$
\gamma \geq \frac{1}{2}, \quad \beta \geq \frac{1}{4}\left(\frac{1}{2}+\gamma\right)^{2}
$$

For $(\gamma, \beta)=(1 / 2,1 / 6)$ we recover the average acceleration method; for $(\gamma, \beta)=(1 / 2,0)$ we obtain the central differences method (although it is not strictly explicit), which is conditionally stable; and for $(\beta, \gamma)=(1 / 4,1 / 2)$ we get the trapezoidal rule, which is unconditionally stable and second-order accurate. In the Newmark scheme, equation (65) is left untouched.

However, numerical damping to prevent the amplification of high-frequency modes cannot be introduced in the Newmark algorithm without degrading the order of accuracy to first-order. There are a number of numerical schemes that generalize the Newmark scheme in order to include maximal dissipation of high frequency modes and minimal of low frequency modes and at the same time maintaining second-order accuracy. In particular: the Hilber- $\alpha$ method [58], the Bossak- $\alpha$ method [59], and the Generalized- $\alpha$ method [60]. We present here the last one, which includes, for certain values of the parameters, the other methods. The Generalized- $\alpha$ method can be seen as a generalization of Newmark's algorithm in the sense that equations (78) are also assumed by this evolution scheme. The generalization takes place when we discrete the set of ODEs given in equation [65]. Let $\left(\boldsymbol{\Psi}_{n}, \dot{\Psi}_{n}, \ddot{\mathbf{\Psi}}_{n}\right)$ be the values of our unknowns and their time derivatives at a time $t=t_{n}$. Then, the discretization of (65) used in the Generalized- $\alpha$ method is given by

$$
\mathbb{M} \cdot \ddot{\Psi}_{n+1-\alpha_{m}}+\mathbb{G} \cdot \dot{\Psi}_{n+1-\alpha_{f}}+\mathbb{K} \cdot \Psi_{n+1-\alpha_{f}}=\boldsymbol{F}_{n+1-\alpha_{f}},
$$

where

$$
\begin{gathered}
\ddot{\mathbf{\Psi}}_{n+1-\alpha_{m}}=\left(1-\alpha_{m}\right) \ddot{\mathbf{\Psi}}_{n+1}+\alpha_{m} \ddot{\mathbf{\Psi}}_{n}, \\
\dot{\boldsymbol{\Psi}}_{n+1-\alpha_{f}}=\left(1-\alpha_{f}\right) \dot{\boldsymbol{\Psi}}_{n+1}+\alpha_{f} \dot{\boldsymbol{\Psi}}_{n}, \\
\boldsymbol{\Psi}_{n+1-\alpha_{f}}=\left(1-\alpha_{f}\right) \boldsymbol{\Psi}_{n+1}+\alpha_{f} \boldsymbol{\Psi}_{n} \\
\boldsymbol{F}_{n+1-\alpha_{f}}=\left(1-\alpha_{f}\right) \boldsymbol{F}_{n+1}+\alpha_{f} \boldsymbol{F}_{n},
\end{gathered}
$$

where $\alpha_{f}$ and $\alpha_{m}$ are constants. The Newmark method corresponds to $\left(\alpha_{f}, \alpha_{m}\right)=(0,0)$, the Hilber $-\alpha$ method to $\alpha_{m}=0$, and the Bossak- $\alpha$ method to $\alpha_{f}=0$. Introducing equations (78) and (79) into equation (81) and rearranging the different terms, we arrive at the following equation for $\ddot{\mathbf{\Psi}}_{n+1}$ :

$$
\begin{aligned}
{\left[\left(1-\alpha_{m}\right) \mathbb{M}\right.} & \left.+\left(1-\alpha_{f}\right) \gamma \Delta t \mathbb{G}+\left(1-\alpha_{f}\right) \beta(\Delta t)^{2} \mathbb{K}\right] \cdot \ddot{\Psi}_{n+1}=\left(1-\alpha_{f}\right) \boldsymbol{F}_{n+1}+\alpha_{f} \boldsymbol{F}_{n}-\alpha_{m} \mathbb{M} \cdot \ddot{\boldsymbol{\Psi}}_{n} \\
& -\mathbb{G} \cdot\left[\dot{\boldsymbol{\Psi}}_{n}+\left(1-\alpha_{f}\right)(1-\gamma) \Delta t \ddot{\boldsymbol{\Psi}}_{n}\right]-\mathbb{K} \cdot\left\{\boldsymbol{\Psi}_{n}+\left(1-\alpha_{f}\right) \Delta t\left[\dot{\boldsymbol{\Psi}}_{n}+\left(\frac{1}{2}-\beta\right) \Delta t \ddot{\boldsymbol{\Psi}}_{n}\right]\right\}
\end{aligned}
$$

Then, the algorithm that we are going to use to solve these equations for our unknowns goes as follows: (i) We solve (86) for $\ddot{\mathbf{\Psi}}_{n+1}$, (ii) We compute $\dot{\mathbf{\Psi}}_{n+1}$ from (78) and, (iii) We compute $\boldsymbol{\Psi}_{n+1}$ from (79). Excepting in very special cases, the method that comes out of this algorithm is implicit. In general implicit schemes are computationally expensive, but since we are using one-dimensional linear elements the matrices that we are dealing with are symmetric tridiagonal and therefore, one can use fast routines to invert them (see, e.g. 61]).

The convergence and stability properties of these algorithms and their high-frequency damping capabilities can be analyzed by casting the time discretization of our ODEs in the form: $\boldsymbol{U}_{n+1}=\mathbb{A} \cdot \boldsymbol{U}_{n}+\boldsymbol{R}_{n}$, and then to 
TABLE I: Values of the coefficients $\left(\alpha_{m}, \alpha_{f}, \beta, \gamma\right)$ that characterize the different evolution algorithms, in order to achieve consistency, stability, and favorable high-frequency mode damping properties.

\begin{tabular}{ccccc}
\hline \hline Algorithm & $\alpha_{m}$ & $\alpha_{f}$ & $\beta$ & $\gamma$ \\
\hline Newmark & 0 & 0 & $\left(1+\rho_{\infty}\right)^{2}$ & $\frac{3-\rho_{\infty}}{2\left(1+\rho_{\infty}\right)}$ \\
Bossak- $\alpha$ & $\frac{\rho_{\infty}-1}{\rho_{\infty}+1}$ & 0 & $\frac{1}{4}\left(1-\alpha_{m}\right)^{2}$ & $\frac{1}{2}-\alpha_{m}$ \\
Hilber- $\alpha$ & 0 & $\frac{1-\rho_{\infty}}{1+\rho_{\infty}}$ & $\frac{1}{4}\left(1+\alpha_{f}\right)^{2}$ & $\frac{1}{2}+\alpha_{f}$ \\
Generalized- $\alpha$ & $\frac{2 \rho_{\infty}-1}{\rho_{\infty}+1}$ & $\frac{\rho_{\infty}}{1+\rho_{\infty}}$ & $\frac{1}{4}\left(1-\alpha_{m}+\alpha_{f}\right)^{2}$ & $\frac{1}{2}-\alpha_{m}+\alpha_{f}$ \\
\hline \hline
\end{tabular}

analyze the truncation error when $\boldsymbol{U}_{n}$ is substituted by the exact expression and the spectral properties of the so-called amplification matrix $\mathbb{A}$ (see, e.g. 54]). A quantity that plays an important role is the spectral radius

$$
\rho_{\infty}=\lim _{\Delta t / T \rightarrow \infty} \rho(\mathbb{A})
$$

where $\rho(\mathbb{A})$ is the spectral radius of the matrix $\mathbb{A}, \Delta t$ is the time step, and $T$ is the vibration period of a generic mode of the system. For $\rho_{\infty}=1$ there is no damping, and the lower $\rho_{\infty}$ is, the bigger the damping gets. In table $\llbracket$ we show the values of the time integration parameters $\left(\alpha_{m}, \alpha_{f}, \beta, \gamma\right)$ for optimal damping properties in terms of the spectral radius $\rho_{\infty}$ (see [54, 62] for details).

The numerical method we use to integrate the ODEs for the motion of the particle, equations (A4A5), is the Bulirsch-Stoer extrapolation method (63, 64]) as described by 65] and [66] (see also [61]).

\section{Structure and Motion of the Mesh}

In the numerical simulations we have carried out we have used different mesh structures, all motivated by the fact that the size of the particle is very small compared to the length scale of the black hole. The features that distinguish these different mesh structures are the following: (i) Refinement. Whether the size of the elements changes along the mesh in order to increase the resolution around the particle. (ii) Particle's location. Whether the particle is located at the position of a node or, in contrast, it is located in the interior of an element. (iii) Motion of the Mesh. Whether the mesh is changing in time (in such a way that the part of the mesh with more resolution always contains the particle) or it is static.

In the case without refinement, we just divide the mesh into a given number of elements, say $N_{o}$, with the same size: $d_{i}=d$, for all $i$. In the case where the particle is located at a node, since the location of the boundaries is also given, at least one element must have a size different from $d$. The way in which we refine the mesh to increase the resolution around the particle is by dividing a certain number of elements in the proximity of the particle a certain number of times. Each time, we divide each of the elements selected into two elements of equal size. For the case in which the particle is at a node, we divide into two a given number of elements, say $p_{\bullet}$, to the right and to the left of the particle. We repeat this a given number of times, say $q_{\text {. }}$. When the particle is located in the interior of a given element, we do the same but with the elements to the right and to the left of the element where the particle is located. In addition, we bisect the element where the particle is located $q_{\bullet}$ times. Then, the mesh is determined by the three parameters $\left(N_{o}, p_{\bullet}, q_{\bullet}\right)$. The total number of elements in the case where the particle is located at a node is: $N_{T}=N_{o}+2 p_{\bullet} q_{\bullet}$, and in the case where it is located in the interior of an element is given by $N_{T}=N_{o}+2 p_{\bullet} q_{\bullet}+2^{q} \bullet-1$. We show an example of these constructions in Figure 4

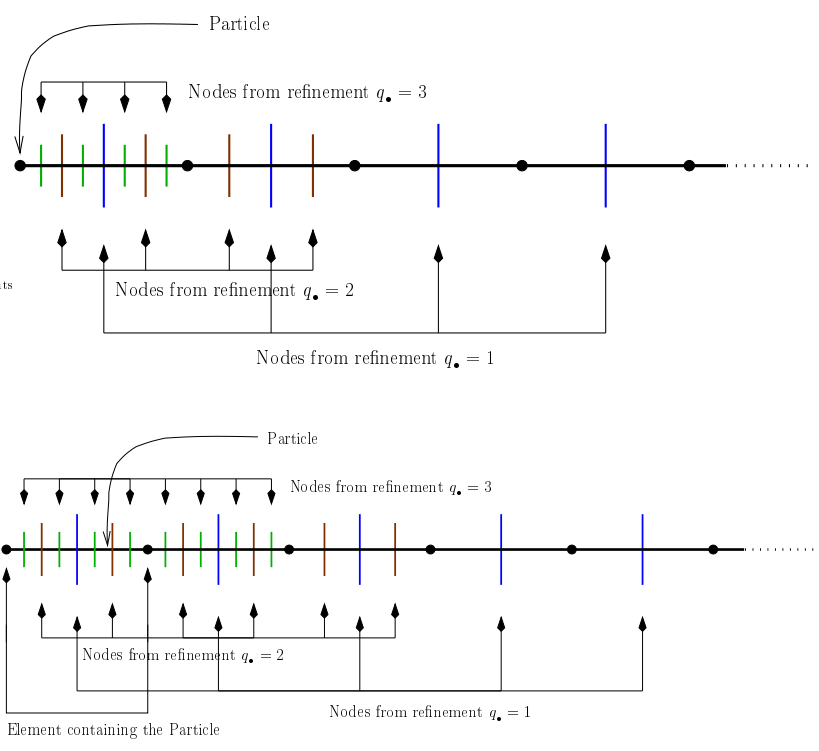

FIG. 4: Examples showing the structure of the Mesh for $\left(p_{\bullet}, q_{\bullet}\right)=(4,3)$ : On the top we have the case of a Mesh where the particle is located at a node. On the bottom we have the case of a Mesh where the particle is always in the interior of an element.

Since the particle is moving, it may be convenient to adapt the mesh so that the finest region is always around the particle. We do this by just translating the structure of the mesh but without modifying it, excepting for the elements containing boundary nodes, whose size we need to change so that after the movement, the mesh fits in our domain. In other words, the resulting mesh is the result of applying a translation to all nodes and the translation distance is just the distance the particle has moved. After the mesh has been moved, we need to find the new associated nodal functions by using the FE interpolation. This is a very simple way of implementing a moving mesh technique, but given that in this problem we know at every moment where the resolution is required we do not need anything more sophisticated, at least at this stage. 


\section{RESULTS FROM THE SIMULATIONS}

We have designed a numerical code with the ingredients described above. To check its performance we have carried out a number of different test. First of all, we have tested it with Gaussian profiles propagating in flat space (both from rest and with initial velocities), and also with Gaussian profiles scattering off the potential of axial and polar modes. To that end we used uniform meshes and the following evolution schemes: the average acceleration method, and the Bossak- $\alpha$, Hilber- $\alpha$, and Generalized- $\alpha$ methods with different values of $\rho_{\infty}$. In all these tests we found stable and second-order convergent (in the $L^{2}$ norm) evolutions. Deviations from second-order convergence were found to be of the order of $0.1 \%$.

When we introduce the point-like object we are introducing source terms that contain a Dirac delta term and a term containing the first derivative of the Dirac delta distribution. These are singular terms, the first one induces a discontinuity in the first derivative of the solution, whereas the second one induces a discontinuity in the solution itself (see the description of these discontinuities given above in Section (I). This loss of smoothness of the solution with respect to the case without particle is quite significant. The presence of Dirac delta distributions can still be handled by the FEM without losing the convergence properties of the algorithms, but the inclusion of source terms with the first derivative of the Dirac delta is too severe to maintain the accuracy and convergence properties of the numerical algorithms (see [53] for a discussion of this issue). As a consequence, the convergence in general drops from second to first order. There is however a way of preserving second-order convergence, consisting of locating the particle at a node and, instead of solving the equation with the force term due to the particle, we solve the equation without the force term in the region to the left and in the region to the right of the particle location using boundary conditions at the particle location that enforce the magnitude of the discontinuities of the solution that the particle source terms dictate. The way of computing these discontinuities is to use the equations that govern their behaviour [equations (43) and (44)]. However, this way of proceeding has some drawbacks depending on the way we implement it. Either it requires to change the structure of the matrices in the FEM discretization of the equation, transforming the linear algebra problem and making it similar to the one that we would get if we were using high-order elements, or it changes the stability properties of our time-evolution schemes from being unconditionally stable (they are implicit schemes) to be subject to a Courant-Friedrichs-Levy stability condition. Moreover, locating the particle at a node means to change the mesh structure at every step in the evolution (excepting for circular orbits), which means to use the FEM interpolation every single time step. The conclusion one can extract from this discussion is that each of the different possible ways in which we can carry out the computations have some advantages and some disvantages. The performance of each of these possible computational schemes depends on the physical setup we want to simulate and therefore, one has analyze on a case by case basis which is the appropiate method to use.

With regard to the choice of the time scheme, which in our framework is equivalent to the choice of the parameters $\left(\beta, \gamma, \alpha_{m}, \alpha_{f}\right)$, the numerical experiments we have performed tell us that the inclusion of the particle generates high-frequency modes that corrupt the solution and therefore we have to choose $\rho_{\infty}$ different from unity to damp those unphysical modes. In the case of the Newmark scheme, this means to loose second-order convergence, and therefore it is not the best choice. Moreover, from our numerical experiments we observe that the Bossak- $\alpha$ scheme is the one that seems to work better in the sense that it is the scheme that damps the high-frequency modes in a more efficient way. The other schemes seems to require a lower $\rho_{\infty}$ (higher damping) than the Bossak- $\alpha$ method for the same performance. We have also seen that the optimal value of $\rho_{\infty}$ to be used depends on the physical case we want to simulate, circular orbits are the ones that require less damping whereas highly eccentric seems to require much more damping (for a comparable pericenter distance). It also depends on whether we move the mesh or not and on the resolution we use.

In order to further assess the capabilities of our computational framework and its adequacy for the type of physical computations we are interested in, we have compared our numerical simulation with different results in the literature for different types of orbits (geodesics). The initial data for the master functions is zero data, that is, $\Psi\left(t_{o}, r\right)=\dot{\Psi}\left(t_{o}, r\right)=0$. This creates an initial burst of spurious radiation which, after suficient time, leaves the computational domain. The global spatial resolution we have used in the simulation varies from $\Delta x=0.1 M$ to $\Delta x=0.02 M$, and the number of times that we refine around the particle goes from $q_{\bullet}=0$ to $q_{\bullet}=10$ (see subsection आID). The physical observers or detectors of the gravitational radiation are located at a tortoise coordinate in the range $\left|r_{*}\right|=2000-2500 M$, and the boundaries are located at a distance in the range $\left|r_{*}\right|=4000-6000 M$. Regarding the time step, it is important to remark that because our evolution algorithms are implicit and unconditionally stable we are not subject to a Courant-Friedrich-Levy type condition on $\Delta t$ (excepting in the case where we use the scheme in which we impose the discontinuities generated by the particle at a given node), which we have taken to be $\Delta t=0.1 \mathrm{M}$.

To begin with, we compare results for circular orbits with the frequency domain code by Poisson [67, 68] (as quoted in 38]), and with the time-domain calculations by Martel [38] (using a formulation based on the ReggeWheeler gauge and solving for the master functions) and Barack and Lousto [39] (using a formulation based on the Lorentz gauge and solving directly for the metric perturbations). The circular orbits considered have a radius 
$p M$ with $p=7.9456$ and our observer is located in the radiation zone at $r_{*}=2000 M$. We compare results for the energy and angular momentum luminosities to infinity with the results of [67, 68] and [38] in Tableஹ] and for the energy luminosities to infinity with the results of [39] in Table III]

We have also compared results for elliptic orbits with the frequency-domain calculations of Cutler et al. 69. and with the time-domain calculations of Martel [38]. We have considered two types of elliptic orbits with orbital parameters given by $(p, e)=(7.50478,0.188917)$ and $(p, e)=(8.75455,0.764124)$. For these orbits we have computed the averaged energy and angular momentum luminosities. The average is taken over a certain number of radial periods and our observer is located at $r_{*}=2500 M$. The results are shown in Table IV

We also compare results for parabolic orbits with the time-domain calculations of Martel [38]. This type of orbits have $e=1$ and are only characterized by the pericenter distance, which is given by $p M / 2$ with $p>8$. As $p$ approaches 8 the number of orbital periods $\left(\Delta \varphi_{p} / 2 \pi\right)$ diverges and the motion shows the so-called zoom-whirl behaviour (see, e.g. 38]), meaning that for a radial period the particle orbits close to the $\mathrm{MBH}$ for a number of orbital periods producing a very characteristic signal (see Figure 7) with a number of cycles that depends on how close $p$ is to 8 . They are therefore a good test bed for the numerical computations. In Table $\nabla$ we show the computations of the total energy and angular momentum radiated to infinity $\left(E^{\infty}, L^{\infty}\right)$ and into the horizon $\left(E^{H}, L^{H}\right)$ for parabolic orbits with $p=8.00001$ and $p=8.001$. For these computations, our observers are located at $r_{*}=-2500 M$ and $r_{*}=2500 M$.

We finish this section by commenting on the waveforms obtained from our numerical computations. We have already mentioned that one of the advantages of the timedomain approach is that it can provide reliable waveforms for a reasonable computational cost. We show that this is indeed the case by plotting the following components of the waveforms: $\Psi_{2,2}^{\mathrm{ZM}}$ for circular orbits with $p=7.9456$, $\Psi_{2,1}^{\mathrm{CPM}}$ for elliptic orbits with $(e, p)=(0.764124,8.75455)$, and $\Psi_{2,2}^{\mathrm{ZM}}$ for parabolic orbits with $p=8.001$ in Figures [5] 6] and [7respectively. To achieve a high degree of smoothness in the waveforms, the damping of the spourious high-frequency modes in the evolution is crucial. In this sense, our simulations show that the evolution numerical algorithms proposed in this paper are suitable for the production of reliable waveforms.

\section{CONCLUSIONS AND DISCUSSION}

In this paper we have presented a new method for computing the gravitational radiation emitted by a point like object orbiting a non-rotating black hole. We have shown that the method is accurate by comparing it with previous results in the literature obtaining an agreement with relative errors of the order of $1 \%$, in many cases even of

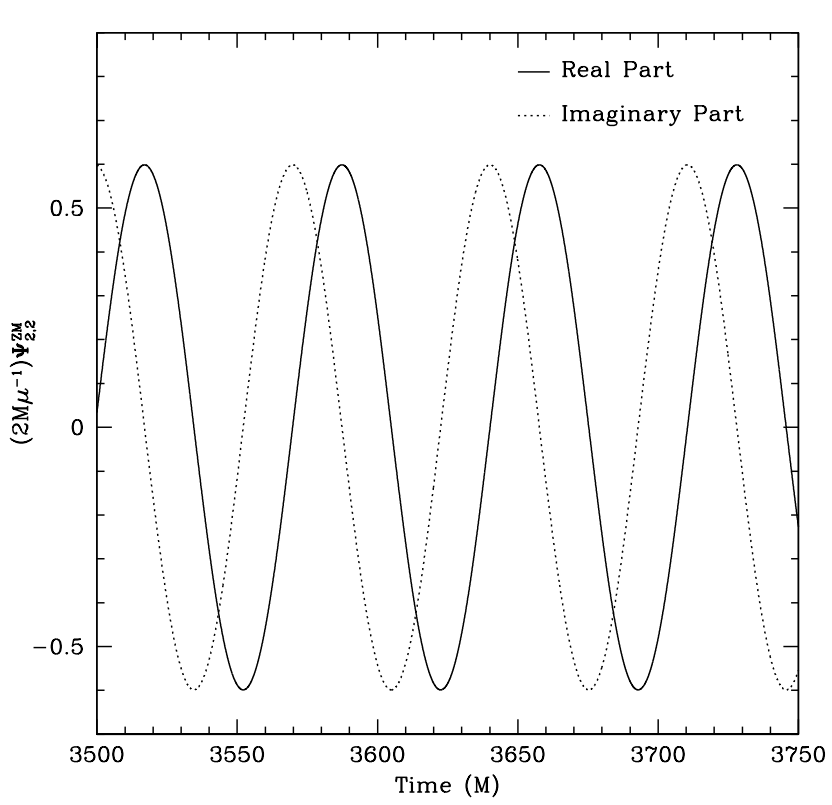

FIG. 5: Component $(\ell, m)=(2,2)$ of the waveform corresponding to circular orbits $(e=0)$ with $p=7.9456$.

the order of $0.1 \%$ or below. We also have shown that these numerical techniques provide sufficiently smooth waveforms, which is one of the goals of these calculation in relation with gravitational-wave data analysis efforts. These results together with the particular feature of the computational method presented suggest that it is a suitable method to be use in self-force calculations for inspiralling EMRBs and in the posterior waveform calculations at the next perturbative order. Our numerical calculations are based on the FEM and related techniques. The main features of the FEM that makes it suitable for the study of EMRBs, and perhaps also for problems that Numerical Relativity deals with, are the following: (i) Proper description of the Computational Domain. This is particularly relevant when we want to solve the perturbative equations in a 2D or 3D setup (see 20]), as it is the case if we want to consider a rotating Kerr black hole, which is the astrophysically relevant case. It would be also relevant for the study of black hole spacetimes in Numerical Relativity. In this scenario, the spacetime geometry may involve holes (inner boundaries arising from black hole singularity excision) and we may wish to use a spherical-type outer boundary to allow gravitational radiation leave the domain smoothly. All these geometric issues have usually caused a number of problems in Finite Differences techniques, but can be handled in a natural way using the FEM. In this respect, the FEM has already shown its capabilities in solving problems in other scientific areas that involve much more complicated domains than the ones we can face in General Relativity. (ii) Imposition of Boundary Conditions. In close connection with the previous point, the underlaying phi- 
TABLE II: Comparison of the computations of energy and angular momentum luminosities at infinity for circular orbits with $p=7.9456$ with results obtained with the frequency-domain code by Poisson [67, 68] and the time-domain code by Martel 38]. They are calculated at $r_{*}=2000 M$. The energy luminosities are expressed in units of $(M / \mu)^{2}$ and the angular momentum luminosities in units of $M / \mu^{2}$. In square brackets we have included the absolute relative difference (rounded to the largest value).

\begin{tabular}{|c|c|c|c|c|c|c|}
\hline$(\ell, m)$ & $\dot{E}_{\ell m}^{\infty}$ & $L_{\ell m}^{\infty}$ & $\dot{E}_{\ell m}^{\infty}[67,68]$ & $\dot{L}_{\ell m}^{\infty}[67,68]$ & $\dot{E}_{\ell m}^{\infty}[38]$ & $\dot{L}_{\ell m}^{\infty}[38]$ \\
\hline$(2,1)$ & $8.1662 \cdot 10^{-7}$ & $1.8289 \cdot 10^{-5}$ & $8.1633 \cdot 10^{-7}[0.04 \%]$ & $1.8283 \cdot 10^{-5}[0.04 \%]$ & $8.1623 \cdot 10^{-7}[0.05 \%]$ & $1.8270 \cdot 10^{-5}[0.1 \%]$ \\
\hline$(2,2)$ & $1.7064 \cdot 10^{-4}$ & $3.8219 \cdot 10^{-3}$ & $1.7063 \cdot 10^{-4}[0.006 \%]$ & $3.8215 \cdot 10^{-3}[0.01 \%]$ & $1.7051 \cdot 10^{-4}[0.08 \%]$ & $3.8164 \cdot 10^{-3}[0.2 \%]$ \\
\hline$(3,2)$ & $2.5204 \cdot 10^{-7}$ & $5.6450 \cdot 10^{-6}$ & $2.5199 \cdot 10^{-7}[0.02 \%]$ & $5.6439 \cdot 10^{-6}[0.02 \%]$ & $2.5164 \cdot 10^{-7}[0.2 \%]$ & $5.6262 \cdot 10^{-6}[0.4 \%]$ \\
\hline$(3,3)$ & $2.5475 \cdot 10^{-5}$ & $5.7057 \cdot 10^{-4}$ & $2.5471 \cdot 10^{-5}[0.02 \%]$ & $5.7048 \cdot 10^{-4}[0.02 \%]$ & $2.5432 \cdot 10^{-5}[0.2 \%]$ & $5.6878 \cdot 10^{-4}[0.4 \%]$ \\
\hline$(4,3)$ & $5.7765 \cdot 10^{-8}$ & $1.2937 \cdot 10^{-6}$ & $5.7751 \cdot 10^{-8}[0.03 \%]$ & $1.2934 \cdot 10^{-6}[0.03 \%]$ & $5.7464 \cdot 10^{-8}[0.6 \%]$ & $1.2933 \cdot 10^{-6}[0.03 \%]$ \\
\hline$(4,4)$ & $4.7270 \cdot 10^{-6}$ & $1.0586 \cdot 10^{-4}$ & $4.7256 \cdot 10^{-6}[0.03 \%]$ & $1.0584 \cdot 10^{-4}[0.02 \%]$ & $4.7080 \cdot 10^{-6}[0.4 \%]$ & $1.0518 \cdot 10^{-4}[0.7 \%]$ \\
\hline$(5,1)$ & $1.2607 \cdot 10^{-15}$ & $2.8237 \cdot 10^{-14}$ & $1.2594 \cdot 10^{-15}[0.1 \%]$ & $2.8206 \cdot 10^{-14}[0.1 \%]$ & $1.2544 \cdot 10^{-15}[0.5 \%]$ & $2.8090 \cdot 10^{-14}[0.6 \%]$ \\
\hline$(5,2)$ & $2.7909 \cdot 10^{-12}$ & $6.2509 \cdot 10^{-11}$ & $2.7896 \cdot 10^{-12}[0.05 \%]$ & $6.2479 \cdot 10^{-11}[0.05 \%]$ & $2.7587 \cdot 10^{-12}[1.2 \%]$ & $6.1679 \cdot 10^{-11}[1.4 \%]$ \\
\hline$(5,3)$ & $1.0936 \cdot 10^{-9}$ & $2.4494 \cdot 10^{-8}$ & $1.0933 \cdot 10^{-9}[0.03 \%]$ & $2.4486 \cdot 10^{-8}[0.04 \%]$ & $1.0830 \cdot 10^{-9}[1.0 \%]$ & $2.4227 \cdot 10^{-8}[1.0 \%]$ \\
\hline
\end{tabular}

TABLE III: Comparison of the computations of energy luminosities [expressed in units of $(M / \mu)^{2}$ ] at infinity for circular orbits with $p=7.9456$ with results obtained in the time domain by Barack and Lousto [39]. They are calculated at $r_{*}=2000 M$. In square brackets we have included the absolute relative difference (rounded to the largest value).

\begin{tabular}{c|c|c}
\hline \hline$(\ell, m)$ & $\dot{E}_{\ell m}^{\infty}$ & $\dot{E}_{\ell m}^{\infty}[39]$ \\
\hline$(2,1)$ & $8.1662 \cdot 10^{-7}$ & $8.1654 \cdot 10^{-7}[0.01 \%]$ \\
$(2,2)$ & $1.7064 \cdot 10^{-4}$ & $1.7061 \cdot 10^{-4}[0.02 \%]$ \\
$(3,1)$ & $2.1732 \cdot 10^{-9}$ & $2.1734 \cdot 10^{-9}[0.01 \%]$ \\
$(3,2)$ & $2.5204 \cdot 10^{-7}$ & $2.5207 \cdot 10^{-7}[0.02 \%]$ \\
$(3,3)$ & $2.5475 \cdot 10^{-5}$ & $2.5479 \cdot 10^{-5}[0.02 \%]$ \\
$(4,1)$ & $8.4055 \cdot 10^{-13}$ & $8.3982 \cdot 10^{-13}[0.09 \%]$ \\
$(4,2)$ & $2.5099 \cdot 10^{-9}$ & $2.5099 \cdot 10^{-9}[0.004 \%]$ \\
$(4,3)$ & $5.7765 \cdot 10^{-8}$ & $5.7759 \cdot 10^{-8}[0.01 \%]$ \\
$(4,4)$ & $4.7270 \cdot 10^{-6}$ & $4.7284 \cdot 10^{-6}[0.03 \%]$ \\
$(5,1)$ & $1.2607 \cdot 10^{-15}$ & $1.2598 \cdot 10^{-15}[0.07 \%]$ \\
$(5,2)$ & $2.7909 \cdot 10^{-12}$ & $2.7877 \cdot 10^{-12}[0.12 \%]$ \\
$(5,3)$ & $1.0936 \cdot 10^{-9}$ & $1.0934 \cdot 10^{-9}[0.02 \%]$ \\
$(5,4)$ & $1.2329 \cdot 10^{-8}$ & $1.2319 \cdot 10^{-8}[0.08 \%]$ \\
$(5,5)$ & $9.4616 \cdot 10^{-7}$ & $9.4623 \cdot 10^{-7}[0.008 \%]$ \\
\hline Total & $2.0293 \cdot 10^{-4}$ & $2.0291 \cdot 10^{-4}[0.01 \%]$ \\
\hline \hline
\end{tabular}

losophy in the FEM is that one should use the mesh that adapts best to the geometric characteristics of the problem we want to solve. In particular, to the boundary conditions, since it is not equally simple and convenient to impose outgoing radiation conditions in a rectangular

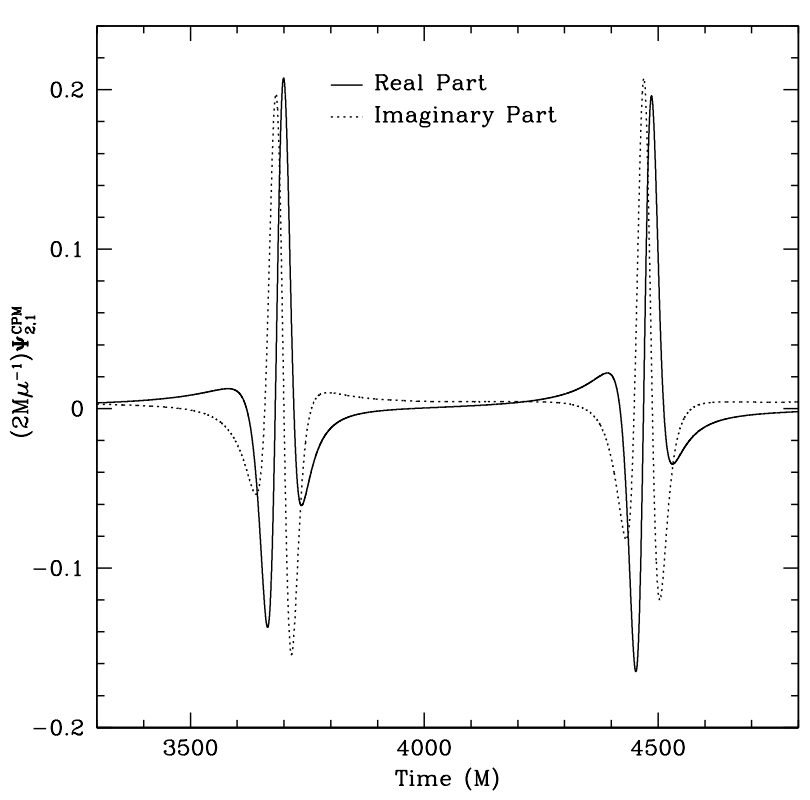

FIG. 6: Component $(\ell, m)=(2,1)$ of the waveform corresponding to elliptic orbits with $e=0.764124$ and $p=8.75455$.

boundary than in a spherical one. This also has an impact when we perform the FEM discretization, since it is based on the weak form of our equations, which can have built in the the boundary conditions. In the case of problems in $2 \mathrm{D}$ or higher dimensions, if the boundary is 
TABLE IV: Computations of the total average energy [in units of $(M / \mu)^{2}$ ] and angular momentum luminosities [in units of $\left.M / \mu^{2}\right],\left\langle\dot{E}^{\infty}\right\rangle$ and $\left\langle\dot{L}^{\infty}\right\rangle$, for elliptic orbits. They are calculated at $r_{*}=2500 M$. We compare with the results obtained by Cutler et al. 69] using a frequency-domain numerical code and by Martel 38] using a time-domain numerical code. We consider two different types of elliptic orbits: Orbit $A:(p, e)=(7.50478,0.188917)$. Orbit $B:(p, e)=(8.75455,0.764124)$.

\begin{tabular}{c|c|c|c|c|c|c}
\hline \hline Orbit & $\left\langle\dot{E}^{\infty}\right\rangle$ & $\left\langle\dot{L}^{\infty}\right\rangle$ & $\left\langle\dot{E}^{\infty}\right\rangle[69]$ & $\left\langle\dot{L}^{\infty}\right\rangle[69]$ & $\left\langle\dot{E}^{\infty}\right\rangle[38]$ & $\left\langle\dot{L}^{\infty}\right\rangle[38]$ \\
\hline$A$ & $3.1640 \cdot 10^{-4}$ & $5.9555 \cdot 10^{-3}$ & $3.1680 \cdot 10^{-4}[0.2 \%]$ & $5.9656 \cdot 10^{-3}[0.2 \%]$ & $3.1770 \cdot 10^{-4}[0.5 \%]$ & $5.9329 \cdot 10^{-3}[0.4 \%]$ \\
$B$ & $2.1004 \cdot 10^{-4}$ & $2.7505 \cdot 10^{-3}$ & $2.1008 \cdot 10^{-4}[0.02 \%]$ & $2.7503 \cdot 10^{-3}[0.01 \%]$ & $2.1484 \cdot 10^{-4}[2.3 \%]$ & $2.7932 \cdot 10^{-3}[1.6 \%]$ \\
\hline \hline
\end{tabular}

TABLE V: Computations of the total energy [in units of $M / \mu^{2}$ ] and angular momentum [in units of $\mu^{-2}$ ] radiated, both to infinity $\left(E^{\infty}, L^{\infty}\right)$ and into the horizon $\left(E^{H}, L^{H}\right)$, in parabolic orbits $(e=1)$. They are calculated at $r_{*}=-2500 M$ and $r_{*}=2500 M$. In square brackets we have included the absolute relative difference (rounded to the largest value) with respect the results obtained by Martel [38] using a time-domain numerical code.

\begin{tabular}{c|c|c|c|c}
\hline \hline$p$ & $E^{\infty}$ & $L^{\infty}$ & $E^{H}$ & $L^{H}$ \\
\hline 8.00001 & $3.5603[3.1 \%]$ & $29.415[2.5 \%]$ & $1.8884 \cdot 10^{-1}[0.05 \%]$ & $1.5112[0.7 \%]$ \\
8.001 & $2.2212[2.7 \%]$ & $18.704[2.1 \%]$ & $1.1339 \cdot 10^{-1}[0.7 \%]$ & $9.0783 \cdot 10^{-1}[0.5 \%]$ \\
\hline \hline
\end{tabular}

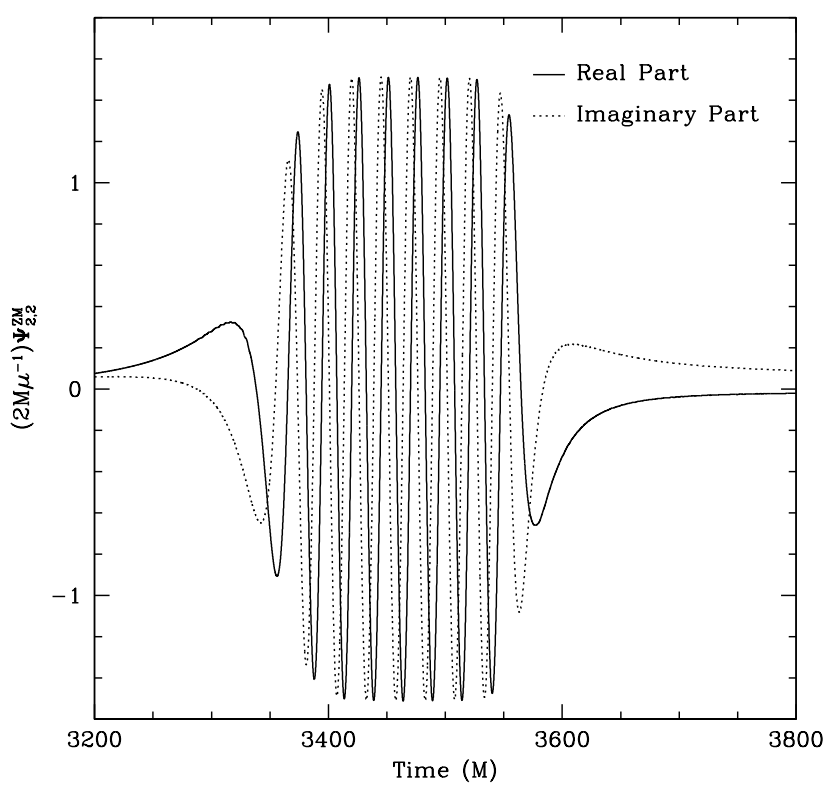

FIG. 7: Component $(\ell, m)=(2,2)$ of the waveforms corresponding to zoom-whirl parabolic orbits $(e=1)$ with $p=$ 8.001 .

natural (adapted to the problem), the implementation of the boundary conditions becomes trivial (see, e.g. [20]). This has advantages even in 1D problems, like the one we have studied in this paper, where the imposition of boundary conditions like Sommerfeld or von Neumann is simpler than in a Finite Differences framework. This paper illustrates this fact. (iii) Treatment of distributions. Many description of EMRBs treat the small body as a point-like object, which despite being somehow unnatural in General Relativity, allows us to perform computa- tions in a consistent way. The consequence of having a point-like object is that the equations that we have to solve contain source terms where Dirac delta distributions and its derivatives (up to second derivatives in the case we were solving the Teukolsky equation sourced by a point-like object) appear. To deal with this kind of distributions in a Finite Difference framework is not an easy task, and the different ways in which one can handle them involved not trivial a priori regularizations of the distributions. Instead, in the FEM, the fact that the discretization is based on the weak form of the equations, an integral formulation, is a key point. We can evaluate the integrals that involve Dirac distributions analytically by using the properties of the distributions, without the need of using any regularization of those distributions. A sample of this has been given in this paper, where we used the weak formulation of the problem to discretize a source term containing the Dirac delta distribution and its first derivative. Then, the type of discretization we would get is in this sense analogous to the one proposed by Price and Lousto [36, 37, 38], where they also used an integral form of the equations to discretize them. Therefore, using the FEM provides an additional advantage for the study of EMRBs where the small object is treated as a point-like object. (iv) Adaptivity. This is a key ingredient for the simulations of EMRBs. The calculations presented in this paper do not necessarily require adaptivity, but they are an excellent benchmark to test these techniques. However, for the case of rotating massive black hole adaptivity may be the only way of performing physically realistic simulations. The FEM is a natural choice to achieve the high level of adaptivity required, both in the construction of the mesh and later by using any of the robust techniques of mesh refinement available (see [20] and references therein).

Apart from these specific reasons, there are other motivations in favour of using the FEM. In this sense it is 
important to mention that because the FEM is based on piecewise (polynomial) approximations, it lies on sound mathematical grounds (see, e.g. [53, 70]). From the point of view of building numerical codes based on the FEM, it is important to emphasize the high degree of independence of the different ingredients of the FEM discretization process [53, 54, 55, 56], which makes it very suitable for modular programming. In addition, the FEM has been widely used in many areas of scientific research and, as a consequence, a number of FEM packages and tools are available for scientific computation.

There are a number of ways of extending this work in order to improve the computational framework in order to simulate EMRBs, and in particular to evalute the radiation-reaction effects. From the computational side we can introduce higher-order elements (by using FE functional spaces with higher-order polynomials), which will improve the accuracy of the computations. From the physical point of view, we can change the description of the gravitational field, meaning the formulation of the perturbative scheme. In this sense, to compute the metric perturbations using the Lorentz gauge, as it has been recently proposed by Barack and Lousto [39], appear to be a very convenient choice for a number of reasons (see [39] for a detailed discussion). Among the advantages of this approach it is worth to mention the following ones: (i) Because one is working with pure metric perturbations the sources do not contain derivatives of the Dirac delta distribution, and hence the solution of the equations is continuous at the particle location, which will improve the accuracy of the computations. (ii) Moreover, in contrast with the computations in the Regge-Wheeler gauge, we do not need a metric perturbation reconstruction procedure (just algebraic computations) to evaluate the self-force. (iii) The regularization procedures to obtain the self-force have only been given in the Lorentz gauge. It also has some disavantages: We need to solve a coupled system of equations instead of single wave-type equations, and there are constraints that need to be satisfied along the evolution.

In the astrophysically motived EMRBs, the $\mathrm{MBH}$ is highly rotating and therefore it is desirable to be able to repeat these calculations by using the Kerr solution as the background spacetime. This is a more difficult problem since it involves three-dimensional PDEs (or twodimensional if we factor out the dependence in the polar angle). In this sense, it is important to mention that the FEM techniques that have been presented and used in this paper can be transfered to the higher-dimensional problem of computing Kerr perturbations. For the same reasons that have been pointed out before, a promising approach may be to solve for metric perturbations of the Kerr black hole in the Lorentz gauge.

Acknowledgements: The authors acknowledge the support of the Center for Gravitational Wave Physics funded by the National Science Foundation under Cooperative Agreement PHY-0114375, and support from NSF grant PHY-0244788 to Penn State University. They also wish to thank the Information Technology Services at Penn State University for the use of the LION-XO computer cluster in some of the calculations presented in this paper, and to Uli Sperhake, Pengtao Sun, and Jinchao $\mathrm{Xu}$ for fruitful discussions.

\section{APPENDIX A: MOTION OF THE POINT-LIKE OBJECT}

To complete the description of our physical problem we have to introduce the equations of motion for the point-like object, which follows the geodesics of the Schwarzschild background black hole spacetime. Then, the four-velocity of the particle satisfies:

$$
u^{\alpha} \nabla_{\alpha} u^{\beta}=0, \quad u^{\alpha}=\frac{d z^{\alpha}(\tau)}{d \tau} .
$$

The static and spherically symmetric character of the background imply the existence of first integrals of the motion (energy and angular momentum), and as it happens in the Newtonian case, the motion takes place on a plane that, without lost of generality, we can take it to be the plane $\theta=\pi / 2\left(u^{\theta}=0\right)$. Then, the equations of motion are equivalent to the following relations:

$$
u^{t}=\frac{E_{p}}{f}, u^{\varphi}=\frac{L_{p}}{r^{2}},\left(u^{r}\right)^{2}=E_{p}^{2}-f\left(1+\frac{L_{p}^{2}}{r^{2}}\right) \text {. }
$$

In order to obtain a well-behaved system of ODEs at the turning points of the radial coordinate $(\dot{r}=0)$ we can use the following alternative quantity

$$
r=\frac{p M}{1+e \cos \chi}
$$

where $e$ denotes the orbital eccentricity and $p$ the semilatus rectum, which can be used as alternative constants of motion to the pair $\left(E_{p}, L_{p}\right)$. Then, the two equations we need to integrate to determine the position of the particle are:

$$
\begin{gathered}
\frac{d \chi}{d t}=\frac{(p-2-2 e \cos \chi)(1+e \cos \chi)^{2} \sqrt{p-6-2 e \cos \chi}}{M p^{2} \sqrt{(p-2)^{2}-4 e^{2}}}, \\
\frac{d \varphi}{d t}=\frac{(\mathrm{A} 4)}{M p^{3 / 2} \sqrt{(p-2)^{2}-4 e^{2}}} .
\end{gathered}
$$

\section{APPENDIX B: GAUSS-LEGENDRE QUADRATURES}

The integrals in the second terms of (68) are computed by using Gauss-Legendre quadratures (see, e.g. [54] 
and $[61])$. Given a function $F(x)$ we approximate its integral over the interval $[a, b]$ by

$$
\int_{a}^{b} d x F(x) \approx \frac{b-a}{2} \sum_{I=1}^{N} W_{I}^{N} F\left(\frac{a+b}{2}+\frac{b-a}{2} u_{I}\right),
$$

where $u_{I}$ is the $I$-th zero of the Legendre polynomial $P_{N}(u)$ (it has exactly $N$ zeros) and $W_{I}^{N}$ are weights as- sociated with the zeros and given by

$$
W_{I}^{N}=\frac{2}{\left(1-u_{I}^{2}\right)\left[P_{N}^{\prime}\left(u_{I}\right)\right]^{2}} \text {. }
$$

An N-point Gauss-Legendre quadrature integrates exactly polynomials of degree $2 N-1$.
[1] L. Barack and C. Cutler, Phys. Rev. 69, 082005 (2004), gr-qc/0310125.

[2] J. R. Gair, L. Barack, T. Creighton, C. Cutler, S. L. Larson, E. S. Phinney, and M. Vallisneri, Class. Quant. Grav. 21, S1595 (2004).

[3] S. Vitale et al., Nucl. Phys. Proc. Suppl. 110, 209 (2002).

[4] K. Danzmann, Advances in Space Research 32, 1233 (2003).

[5] K. Danzmann and A. Rudiger, Class. Quant. Grav. 20, S1 (2003).

[6] T. Prince, American Astronomical Society Meeting 202, 3701 (2003).

[7] E. Poisson, Living Rev. Relativity 7, 6 (2004), URL http://www.livingreviews.org/lrr-2004-6 grqc/0306052.

[8] C. O. Lousto, ed., Gravitational Radiation from Binary Black Holes: Advances in the Perturbative Approach. Special Issue of vol. 22 (Classical and Quantum Gravity, Institute of Physics, London, 2005).

[9] Y. Mino, M. Sasaki, and T. Tanaka, Phys. Rev. D55, 3457 (1997), gr-qc/9606018.

[10] T. C. Quinn and R. M. Wald, Phys. Rev. D56, 3381 (1997), gr-qc/9610053.

[11] S. Detweiler and B. F. Whiting, Phys. Rev. D67, 024025 (2003), gr-qc/0202086.

[12] L. Barack and A. Ori, Phys. Rev. D61, 061502 (2000), gr-qc/9912010.

[13] L. Barack, Phys. Rev. D62, 084027 (2000), grqc/0005042.

[14] L. Barack, Phys. Rev. D64, 084021 (2001), grqc/0105040.

[15] L. Barack, Y. Mino, H. Nakano, A. Ori, and M. Sasaki, Phys. Rev. Lett. 88, 091101 (2002), gr-qc/0111001.

[16] C. O. Lousto, Phys. Rev. Lett. 84, 5251 (2000), grqc/9912017.

[17] C. O. Lousto (2005), astro-ph/0501238.

[18] C. V. Vishveshwara, Phys. Rev. D1, 2870 (1970).

[19] S. Chandrasekhar and S. Detweiler, Royal Society of London Proceedings Series A 344, 441 (1975).

[20] C. F. Sopuerta, P. Sun, P. Laguna, and J. Xu (2005), gr-qc/0507112.

[21] C. T. Cunningham, R. H. Price, and V. Moncrief, ApJ 224, 643 (1978).

[22] T. Regge and J. A. Wheeler, Phys. Rev. 108, 1063 (1957).

[23] F. J. Zerilli, Phys. Rev. Lett. 24, 737 (1970).

[24] V. Moncrief, Ann. Phys. (N.Y.) 88, 323 (1974).

[25] S. A. Teukolsky, Phys. Rev. Lett. 29, 1114 (1972).

[26] S. A. Teukolsky, ApJ 185, 635 (1973).

[27] S. Chandrasekhar, The mathematical theory of black holes (Oxford University Press, New York, 1992).

[28] K. Kokkotas and B. Schmidt, Living Reviews in Relativity 2, 2 (1999).

[29] H.-P. Nollert, Classical and Quantum Gravity 16, 159 (1999).

[30] O. Sarbach and M. Tiglio, Phys. Rev. D64, 084016 (2001), gr-qc/0104061.

[31] K. Martel and E. Poisson, Phys. Rev. D71, 104003 (2005), gr-qc/0502028.

[32] K. Martel, Particles and Black Holes: Time-Domain Integration of the Equations of Black-Hole Perturbation Theory, Ph.D. thesis, The University of Guelph (2003).

[33] A. Nagar and L. Rezzolla, Class. Quant. Grav. 22, R167 (2005), gr-qc/0502064.

[34] F. J. Zerilli, Phys. Rev. D 2, 2141 (1970).

[35] M. Davis, R. Ruffini, and J. Tiomno, Phys. Rev. D 5, 2932 (1972).

[36] C. O. Lousto and R. H. Price, Phys. Rev. D56, 6439 (1997), gr-qc/9705071.

[37] K. Martel and E. Poisson, Phys. Rev. D66, 084001 (2002), gr-qc/0107104.

[38] K. Martel, Phys. Rev. D69, 044025 (2004), grqc/0311017.

[39] L. Barack and C. O. Lousto (2005), gr-qc/0510019.

[40] U. H. Gerlach and U. K. Sengupta, Phys. Rev. D19, 2268 (1979).

[41] U. H. Gerlach and U. K. Sengupta, Phys. Rev. D22, 1300 (1980).

[42] S. Jhingan and T. Tanaka, Phys. Rev. D67, 104018 (2003), gr-qc/0211060.

[43] R. A. Isaacson, Phys. Rev. 166, 1263 (1968).

[44] R. A. Isaacson, Phys. Rev. 166, 1272 (1968).

[45] J. N. Goldberg, A. J. Macfarlane, E. T. Newman, F. Rohrlich, and E. C. G. Sudarshan, J. Math. Phys. 8, 2155 (1967).

[46] A. Bayliss and E. Turkel, Comm. Pure Appl. Math. 33, 707 (1980).

[47] A. Bayliss, M. Gunzburger, and E. Turkel, SIAM J. Appl. Math. 42, 430 (1982).

[48] B. Alpert, L. Greengard, and T. Hagstrom, SIAM J. Numer. Anal. 37, 1138 (2000).

[49] B. Alpert, L. Greengard, and T. Hagstrom, J. Comp. Phys. 180, 270 (2002).

[50] S. R. Lau, J. Comput. Phys. 199, 376 (2004), grqc/0401001.

[51] S. R. Lau, Class. Quant. Grav. 21, 4147 (2004).

[52] S. R. Lau (2005), gr-qc/0507140.

[53] G. Strang and G. J. Fix, An Analysis of the Finite Element Method (Prentice-Hall, Inc., Englewood Cliffs, N.J., 1973). 
[54] T. J. R. Hughes, The Finite Element Method (PrenticeHall, Inc., Englewood Cliffs, N. J., 1987).

[55] I. Babuška and T. Strouboulis, The finite element method and its reliability, Numerical Mathematics and Scientific Computation (Oxford Science Publications, 2001).

[56] O. C. Zienkiewicz, The Finite Element Method (McGraw-Hill, New York, 1977).

[57] N. N. Newmark, Journal of the Engineering Mechanics Division, Proceedings of the A.S.C.E. 85, 67 (1959).

[58] H. M. Hilber, T. J. R. Hughes, and R. L. Taylor, Earthquake Engineering and Structural Dynamics 5, 283 (1977).

[59] W. L. Wood, M. Bossak, and O. C. Zienkiewicz, International Journal for Numerical Methods in Engineering 15, 1562 (1981).

[60] J. Chung and G. Hulbert, Journal of Applied Mechanics, Transactions of the A.S.M.E. 60, 371 (1993).

[61] W. H. Press, B. P. Flannery, S. A. Teukolsky, and
W. T. Vetterling, Numerical Recipes: The Art of Scientific Computing (Cambridge University Press, Cambridge (UK) and New York, 1992).

[62] D. Kuhl and R. Ekkehard, Comput. Methods Appl. Mech. Engrg. 178, 343 (1999).

[63] R. Bulirsch and J. Stoer, Num. Math. 8, 1 (1966).

[64] J. Stoer and R. Bulirsch, Introduction to Numerical Analysis (Springer-Verlag, New York, 1993).

[65] T. Ito and T. Fukushima, AJ 114, 1260 (1997).

[66] T. Fukushima, AJ 112, 1298 (1996).

[67] E. Poisson, Phys. Rev. D52, 5719 (1995), gr-qc/9505030.

[68] E. Poisson, Phys. Rev. D55, 7980 (1997).

[69] C. Cutler, D. Kennefick, and E. Poisson, Phys. Rev. D50, 3816 (1994).

[70] P. G. Ciarlet, The Finite Element Method for Elliptic Problems (North-Holland Publishing Co., AmsterdamNew York-Oxford, 1978). 